\title{
Capacity Limits of Multiuser Multiantenna Cognitive Networks
}

\author{
Yang Li, Student Member, IEEE, and Aria Nosratinia, Fellow, IEEE
}

\begin{abstract}
Unlike point-to-point cognitive radio, where the constraint imposed by the primary rigidly curbs the secondary throughput, multiple secondary users have the potential to efficiently harvest the spectrum and share it among themselves. This paper analyzes the sum throughput of a multiuser cognitive radio system with multiantenna base stations, either in the uplink or downlink mode. The primary and secondary have $N$ and $n$ users, respectively, and their base stations have $M$ and $m$ antennas, respectively. We show that an uplink secondary throughput grows with $\frac{m}{N+1} \log n$ if the primary is a downlink system, and grows with $\frac{N_{m}}{M+1} \log n$ if the primary is an uplink system. These growth rates are shown to be optimal and can be obtained with a simple threshold-based user selection rule. In addition, we show that the secondary throughput can grow proportional to $\log n$, while simultaneously the interference on the primary is forced down to zero, asymptotically. For a downlink secondary, it is shown that the throughput grows with $m \log \log n$ in the presence of either an uplink or downlink primary system. In addition, the interference on the primary can be made to go to zero asymptotically, while the secondary throughput increases proportionally to $\log \log n$. The effect of unequal path loss and shadowing is also studied. It is shown that under a broad class of path loss and shadowing models, the secondary throughput growth rates remain unaffected.
\end{abstract}

Index Terms-Capacity scaling, cognitive radio, interference, multiuser diversity.

\section{INTRODUCTION}

$\mathrm{C}$ URRENTLY, the spectrum assigned to licensed (primary) users is underutilized [1]. Cognitive radio aims to improve the utilization of spectrum by allowing cognitive (secondary) users to access the same spectrum as primary users, as long as performance degradation of the primary users is tolerable.

In general, secondary users can access the spectrum via methods known as overlay, interweave, and underlay [2]. In the overlay technique, the secondary user not only transmits its own signal, but also acts as a relay to compensate for its interference on the primary user. The overlay method depends on the secondary transmitter having access to primary's message [3]. In the interweave technique [4], the secondary user first senses spectrum holes and then transmits in the detected holes. Reliable sensing in the presence of fading and shadowing has

Manuscript received August 18, 2010; revised October 30, 2011; accepted January 18, 2012. Date of publication March 22, 2012; date of current version June 12, 2012. The material in this paper was presented in part at the $2010 \mathrm{In}$ formation Theory and Applications Workshop and the 2011 IEEE Global Communications Conference.

The authors are with the University of Texas at Dallas, Richardson, TX 75080 USA (e-mail: yang@utdallas.edu; aria@utdallas.edu).

Communicated by R. Yates, Associate Editor for Communication Networks.

Color versions of one or more of the figures in this paper are available online at http://ieeexplore.ieee.org.

Digital Object Identifier 10.1109/TIT.2012.2191711 proved to be challenging [5]. Finally, in the underlay technique [6], the secondary can transmit as long as the interference caused on the primary is less than a predefined threshold. The secondary user in this case is neither required to know the primary user's message nor restricted to transmit in spectrum holes.

This paper studies performance limits of an underlay cognitive network consisting of multiuser and multiantenna primary and secondary systems. The primary and secondary systems are subject to mutual interference, where the secondary must comply with a set of interference constraints imposed by the primary. We are interested in the secondary throughput, i.e., the sum rate averaged over channel realizations, as the number of secondary users grows. Moreover, we study how the secondary throughput is affected by the size of primary network as well as the severity of the interference constraints.

A summary of the results of this paper is as follows. We assume that the primary and secondary have $N$ and $n$ users, respectively, and their base stations have $M$ and $m$ antennas, respectively. In this paper, $n$ is allowed to grow (to infinity) while $N, M$, and $m$ are bounded (not scaling with $n$ ).

1) Secondary uplink (MAC): The secondary throughput is shown to grow as $\Theta(\log n)$, which is achieved by a threshold-based user selection rule. More precisely, the throughput of the secondary multiple access channel (MAC) grows as $\frac{m}{N+1} \log n+O(1)$ when it coexists with the primary broadcast channel, and grows as $\frac{m}{M+1} \log n+O(1)$ when it coexists with the primary MAC channel. By developing asymptotically tight upper bounds, these growth rates are further proven to be optimal. Moreover, the interference on the primary system can be asymptotically forced to zero, while the secondary throughput still grows as $\Theta(\log n)$. Specifically, for some nonnegative exponent $q$, the interference on the primary can be made to decline as $\Theta\left(n^{-q}\right)$, while the throughput of a secondary MAC grows as $\frac{m-q N}{N+1} \log n+O(1)$ and $\frac{m-q M}{M+1} \log n+O(1)$, respectively, in cases of primary broadcast and MAC channel. The aforementioned results imply that asymptotically the secondary system can attain a nontrivial throughput without degrading the performance of the primary system.

2) Secondary downlink (broadcast): The secondary throughput is shown to scale with $m \log \log n+O(1)$ in the presence of either the primary broadcast or MAC channel. Hence, the growth rate of throughput is unaffected (thus optimal) by the presence of the primary system. In addition, the interference on the primary can be asymptotically forced to zero, while maintaining the secondary throughput as $\Theta(\log \log n)$. Specifically, for 
an arbitrary exponent $0<q<1$, the interference can be made to decline as $\Theta\left((\log n)^{-q}\right)$, while the secondary throughput grows as $m(1-q) \log \log n+O(1)$.

3) Nonhomogeneous networks: Secondary throughput under nonhomogeneous internode link gains is studied for both secondary MAC and broadcast. It is shown that even if the nodes experience unequal path loss and shadowing, under a broad class of path loss and shadowing models, the secondary throughput growth rates remain unaffected.

Much of the past work in the underlay cognitive radio involves point-to-point primary and secondary systems. Ghasemi and Sousa [6] studies the ergodic capacity of a point-to-point secondary link under various fading channels. Multiple antennas at the secondary transmitter are exploited by Zhang and Liang [7] to manage the tradeoff between the secondary throughput and the interference on the primary. In the context of multiuser cognitive radios, Zhang et al. [8] study the power allocation of a single-antenna secondary system under various transmit power constraints as well as interference constraints. Gastpar [9] studies the secondary capacity via translating a receive power constraint into a transmit power constraint.

Recently, ideas from opportunistic communication [10] have been used in underlay cognitive radios by selectively activating one or more secondary users to maximize the secondary throughput while satisfying interference constraints. The user selection in cognitive radio is complicated because the secondary system must be mindful of two criteria: The interference on the primary and the rate provided to the secondary. Hamdi et al. [11] select secondary users with channels almost orthogonal to a single primary user, so that the interference on the primary is reduced. Jamal et al. [12], [13] obtain interesting scaling results for the throughput by selecting users causing the least interference. Some distinctions of our work and [12], [13] are worth noting. First, Jamal et al. [12], [13] study the hardening of sum rate via convergence in probability, while we analyze the throughput, which requires a very different approach. ${ }^{1}$ Second, we study a multiantenna cognitive network and the effect of the primary network size (number of constraints) on the secondary throughput, whereas Jamal et al. [12], [13] consider a single antenna network with a single primary constraint.

We use the following notation: $[\cdot]_{i, j}$ refers to the $(i, j)$ element in a matrix, $|\cdot|$ refers to the cardinality of a set or the Euclidean norm of a vector, $\operatorname{diag}(\cdot)$ refers to a diagonal matrix, $\operatorname{tr}(\cdot)$ refers to the trace of a matrix, and $I_{k}$ refers to the $k \times k$ identity matrix. All $\log (\cdot)$ is natural base. For any $\epsilon>0$, some positive $c_{1}$ and $c_{2}$, and sufficiently large $n$ :

$$
\begin{aligned}
f(n) & =O(g(n)): & & |f(n)|<c_{1}|g(n)| \\
f(n) & =\Theta(g(n)): & c_{2}|g(n)|<|f(n)| & <c_{1}|g(n)| \\
f(n) & =o(g(n)): & & |f(n)|<\epsilon|g(n)| .
\end{aligned}
$$

\footnotetext{
${ }^{1}$ In general, convergence in probability does not imply convergence in average throughput [14]. For example, consider

$$
X_{n}= \begin{cases}1 & \text { with probability } 1 \frac{1}{n} \\ \exp \left(n^{2}\right) & \text { with probability } \frac{1}{n}\end{cases}
$$

with rates $R_{n}=\log \left(1+X_{n}\right)$. Then, $\lim _{n \uparrow \infty} R_{n}=\log 2$ in probability, however, $\lim _{n \uparrow \infty} \mathbb{E}\left[R_{n}\right]=\infty$ in probability. Therefore, the average rate $\mathbb{E}\left[R_{n}\right]$ may not be predicted based on the hardening (in probability) of $R_{n}$.
}
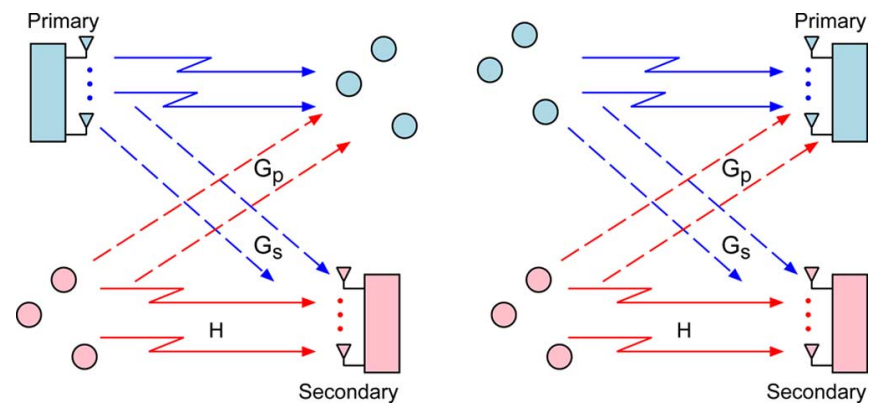

Fig. 1. Coexistence of the secondary MAC channel and the primary system.
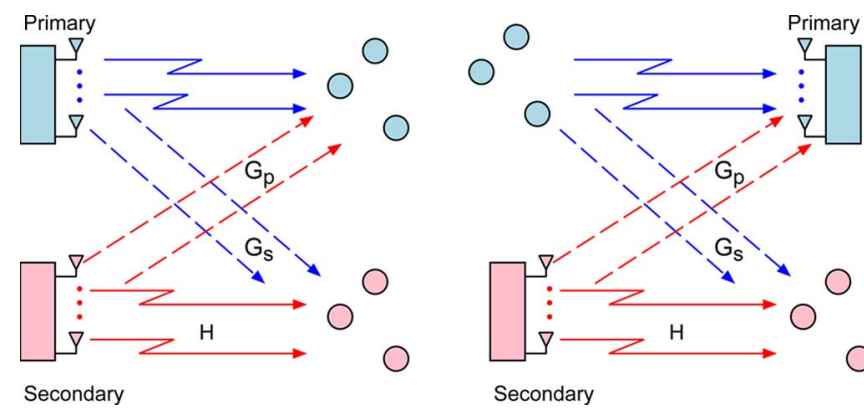

Fig. 2. Coexistence of the secondary broadcast channel and the primary system.

In this paper, we define throughput as the sum rate averaged over all channel realizations. We let $\mathcal{R}_{\text {mac }, w / o}^{\mathrm{opt}}$ and $\mathcal{R}_{b c, w / o}^{\mathrm{opt}}$ be the maximum throughput achieved by the secondary MAC and broadcast channel in the absence of the primary, respectively. In this case, we have regular MAC and broadcast channels, and it is well known that $\mathcal{R}_{\mathrm{mac}, w / o}^{\mathrm{opt}}$ scales as $m \log n[15]$, and $\mathcal{R}_{b c, w / o}^{\mathrm{opt}}$ scales as $m \log \log n[16]$.

The remainder of this paper is organized as follows. Section II describes the system model. The throughput of the secondary MAC channel is studied in Section III, where in Section III-C, we prove the achieved throughout is asymptotically optimal. The average throughput of the secondary broadcast channel is investigated in Section IV. Section V studies the effect of pathloss and shadowing on the secondary throughput. Numerical results are shown in Section VI. Finally, Section VII concludes this paper.

\section{System Model}

We consider a cognitive network consisting of a primary and a secondary, each being either an MAC or broadcast channel (see Figs. 1 and 2). The primary system has one base station with $M$ antennas and $N$ users, while the secondary system consists of one base station with $m$ antennas and $n$ users. The primary and secondary are subject to mutual interference, which is treated as noise (no interference decoding). The secondary system must comply with a set of interference power constraints imposed by the primary. For simplicity of exposition, primary and secondary users are assumed initially to have one antenna, however, as shown in the sequel, most of the results can be directly extended to a scenario where each user has multiple antennas. 
A block-fading channel model is assumed. All channel coefficients are fixed throughout each transmission block, and are independent identically distributed (i.i.d.) circularly-symmetriccomplex-Gaussian with zero mean and unit variance, denoted by $\mathcal{C} \mathcal{N}(0,1)$. The secondary base station acts as a scheduler: For each transmission block, a subset of the secondary users is selected to transmit to (or receive from) the secondary base station. We denote the collection of selected (active) secondary users as $\mathcal{S}$.

We begin by introducing a system model that applies to all four scenarios in Figs. 1 and 2, thus simplifying notation in the remainder of the paper. The secondary received signal is given by

$$
\mathbf{y}=\mathbf{H}(\mathcal{S}) \mathbf{x}_{s}+\mathbf{G}_{s} \mathbf{x}_{p}+\mathbf{w}
$$

where $\mathbf{y}$ represents the received signal vector, either signals at a multiantenna base station (uplink) or at different users (downlink). $\mathbf{H}(\mathcal{S})$ is the channel coefficient matrix between the active secondary users and their base station. $\mathbf{G}_{s}$ represents the crosschannel coefficient matrix from the primary transmitter(s) to the secondary receiver(s). The primary and secondary transmit signal vectors are $\mathbf{x}_{p}$ and $\mathbf{x}_{x}$. The variable $\mathbf{w}$ is the received noise vector, where each entry of $\mathbf{w}$ is i.i.d. $\mathcal{C N}(0,1)$.

We assume both primary and secondary systems use Gaussian signaling, subject to short-term power constraints. The transmit covariance matrices of the primary and secondary systems are

$$
Q_{p}=\mathbb{E}\left[\mathbf{x}_{p} \mathbf{x}_{p}^{\dagger}\right]
$$

and

$$
Q_{s}=\mathbb{E}\left[\mathbf{x}_{s} \mathbf{x}_{s}^{\dagger}\right] .
$$

When the secondary is an MAC channel, each secondary user is subject to an individual short-term power constraint $\rho_{s}$. The users do not cooperate; therefore, $Q_{s}$ is diagonal:

$$
Q_{s}=\operatorname{diag}\left(\rho_{1}, \ldots, \rho_{|\mathcal{S}|}\right)
$$

where $\rho_{\ell} \leq \rho_{s}$, for $\ell=1, \ldots,|\mathcal{S}|$. In this case, $\mathbf{H}(\mathcal{S})$ has dimension $m \times|\mathcal{S}|$.

When the secondary is a broadcast channel, we assume the secondary base station is subject to a short-term power constraint $P_{s}$ :

$$
\operatorname{tr}\left(Q_{s}\right) \leq P_{s}
$$

In this case, $\mathbf{H}(\mathcal{S})$ has dimension $|\mathcal{S}| \times m$. When the primary is an MAC channel, each primary user transmits with power $\rho_{p}$ without user cooperation:

$$
Q_{p}=\rho_{p} I_{N}
$$

Furthermore, each receive antenna at the primary base station can tolerate interference with power $\Gamma$ from the secondary system, ${ }^{2}$ i.e.,

$$
\left[\mathbf{G}_{p} Q_{s} \mathbf{G}_{p}^{\dagger}\right]_{\ell, \ell} \leq \Gamma
$$

${ }^{2}$ If each primary antenna or user tolerates a different interference power, the results of this paper still hold, as seen later. for $\ell=1, \ldots, M$, where $\mathbf{G}_{p}$ represents the cross-channel coefficient matrix from the secondary base station (or active users) to the primary base station.

When the primary is a broadcast channel, the power constraint at the primary base station is $\operatorname{tr}\left(Q_{p}\right) \leq P_{p}$. For simplicity, we assume ${ }^{3}$

$$
Q_{p}=\frac{P_{p}}{M} I_{M}
$$

Furthermore, each primary user tolerates interference with power $\Gamma$ :

$$
\left[\mathbf{G}_{p} Q_{s} \mathbf{G}_{p}^{\dagger}\right]_{\ell, \ell} \leq \Gamma
$$

for $\ell=1, \ldots, N$, where $\mathbf{G}_{p}$ is the cross-channel coefficient matrix from the secondary base station (or active users) to the primary users.

\section{Cognitive MAC ChANNEL}

Consider an MAC secondary in the presence of either a broadcast or MAC primary. We wish to find how much throughput is available to the secondary subject to rigid constraints on the secondary-on-primary interference. We first construct a transmission strategy and find the corresponding (achievable) throughput. Then, we develop upper bounds that are tight with respect to the throughput achieved.

The framework for the transmission strategy is as follows: For each transmission block, the secondary base station determines an active user set $\mathcal{S}$ as well as transmit power for all active users $Q_{s}$. For each transmission, from (1), the sum rate of the secondary system is [17]

$$
\begin{aligned}
R_{\text {mac }}=\log \operatorname{det} & \left(I+\mathbf{H}(\mathcal{S}) Q_{s} \mathbf{H}^{\dagger}(\mathcal{S})+\mathbf{G}_{s} Q_{p} \mathbf{G}_{s}^{\dagger}\right) \\
& -\log \operatorname{det}\left(I+\mathbf{G}_{s} Q_{p} \mathbf{G}_{s}^{\dagger}\right) .
\end{aligned}
$$

subject to the interference constraints (9) and (7) for the primary broadcast and MAC channel, respectively.

The secondary throughput is obtained by averaging $R_{\text {mac }}$ over channel realizations

$$
\mathcal{R}_{\text {mac }}=\mathbb{E}\left[R_{\text {mac }}\right] .
$$

For the development of upper bounds, we assume the secondary base station knows all the channels. This is a genie-like argument that is used solely for development of upper bounds. For the achievable scheme, the requirement is more modest and is outlined after the description of the achievable scheme (see Remark 1).

\section{A. Achievable Scheme}

The objective is to choose $\mathcal{S}$ and $Q_{s}$, i.e., the secondary active transmitters and their power, such that secondary throughput is maximized subject to interference constraints on the primary.

The choice of $\mathcal{S}$ and $Q_{s}$ is coupled through the interference constraints: Either more secondary users can transmit with smaller power, or fewer of them with higher power. We focus on a simple power policy that all active secondary users

\footnotetext{
${ }^{3}$ The asymptotic results remain the same, even if we allow $Q_{p}$ to be an arbitrary covariance matrix.
} 
transmit with the maximum allowed power $\rho_{s}$. Hence, given an active user set $\mathcal{S}$, we have

$$
Q_{s}=\rho_{s} I_{|\mathcal{S}|} .
$$

It will be shown that the ON-OFF transmission (without any further power adaptation) suffices to asymptotically achieve the maximum throughput. Furthermore, its simplicity facilitates analysis.

Recall that each primary user can tolerate interference with power $\Gamma$. The interference on a primary user is guaranteed to be below this level if $k_{s}$ secondary users are active, each causing interference no more than $\alpha=\frac{\Gamma}{k_{s}}$. This bound allows us to honor the interference constraints on the primary while decoupling the action of different secondary users. Based on this observation, we construct a user selection rule as follows. First, we define an eligible secondary user set that disqualifies users that cause too much interference on the primary

$$
\mathcal{A}=\left\{i: \rho_{s}\left|\left[\mathbf{G}_{p}\right]_{j i}\right|^{2}<\alpha, \text { for all } j\right\}
$$

where $\left[\mathbf{G}_{p}\right]_{j i}$ is the channel coefficient from the secondary user $i$ to the primary user (antenna) $j$, and $\alpha$ is a pre-designed interference quota. A secondary user is eligible if its interference on each primary user (antenna) is less than $\alpha$. Now, to satisfy the interference bound, we limit the number of secondary transmitters to no more than $k_{s}$, where

$$
k_{s}=\frac{\Gamma}{\alpha} .
$$

If $|\mathcal{A}| \leq k_{s}$, then all eligible users can transmit. If $|\mathcal{A}|>k_{s}$, then $k_{s}$ users will be chosen randomly from among the eligible users to transmit. ${ }^{4}$

The number of eligible users, $|\mathcal{A}|$, is a random variable; the number of active users is

$$
|\mathcal{S}|=\min \left(k_{s},|\mathcal{A}|\right) .
$$

The transmission of $|\mathcal{S}|$ eligible users induces interference no more than $\Gamma$ on any primary user or antenna. Notice that the manner of user selection guarantees that the channel coefficients in $\mathbf{H}(\mathcal{S})$ remain independent and distributed as $\mathcal{C N}(0,1)$.

Now, we want to design an interference quota $\alpha$ to maximize the secondary throughput. Neither very small nor very large values of $\alpha$ are useful within our framework: If $\alpha$ is very small, for most transmissions few (if any) secondary users will be eligible; thus, the secondary throughput will be small. If $\alpha>\Gamma$, any transmitting user might violate the interference constraint, so the secondary must shut down (equivalently, we have $k_{s}<1$ ). The value of individual interference constraint $\alpha$, or equivalently $k_{s}$, must be set somewhere between these extremes.

Clearly, a desirable outcome would be to allow exactly the number of users that are indeed eligible for transmission, i.e., $k_{s} \approx|\mathcal{A}|$. But one cannot guarantee this in advance because

\footnotetext{
${ }^{4}$ Naturally, the number of active users must be an integer, i.e., $\left\lfloor k_{s}\right\rfloor$. We do not carry the floor operation in the following developments for simplicity, noting that due to the asymptotic nature of the analysis, the floor operation has no effect on the final results.
}

$|\mathcal{A}|$ is a random variable. Motivated by this general insight, we choose $\alpha$ such that

$$
k_{s}=\mathbb{E}[|\mathcal{A}|] .
$$

In Section III-C, we will verify that this choice of $\alpha$ is enough to asymptotically achieve the maximum throughput.

Remark 1: The aforementioned scheme does not require the secondary users to have full channel knowledge. Each secondary user can compare its own cross-channel gains with a predefined interference quota $\alpha$, and then decide its eligibility. After this, each eligible user can inform the secondary base station via 1-bit, so that the secondary base station can determine $\mathcal{A}$ without knowing the cross channels from the secondary users to the primary system. The secondary channels $\mathbf{H}(\mathcal{S})$ and the cross channels $\mathbf{G}_{s}$ can be estimated at the secondary base station. Therefore, this scheme can be implemented with little exchange of channel knowledge.

\section{B. Throughput Calculation}

Secondary MAC With Primary Broadcast: The primary base station transmits to $N$ primary users, where each user tolerates interference with power $\Gamma$. Notice that in (13), $\left[\mathbf{G}_{p}\right]_{j i}$ is the channel coefficient from the secondary user $i$ to the primary user $j$ which is i.i.d. $\mathcal{C N}(0,1)$. Thus, $\left|\left[\mathbf{G}_{p}\right]_{j i}\right|^{2}$ is i.i.d. exponential. Therefore, $|\mathcal{A}|$ is binomially distributed with parameter $(n, p)$, where

$$
p=\left(1-e^{-\frac{\alpha}{\rho_{s}}}\right)^{N} .
$$

From (16), the interference quota $\alpha=\frac{\Gamma}{k_{s}}$ is chosen such that

$$
\begin{aligned}
k_{s} & =n p \\
& =n\left(\frac{\Gamma}{\rho_{s}}\right)^{N} k_{s}^{-N}+O\left(n k_{s}^{-(N+1)}\right) .
\end{aligned}
$$

Denote the associated solution for $k_{s}$ as $\bar{k}_{s}$ :

$$
\bar{k}_{s}=\left(\frac{\Gamma}{\rho_{s}}\right)^{\frac{N}{N+1}}(n)^{\frac{1}{N+1}}+O(1) .
$$

Thus, we can see $\Theta\left(n^{\frac{1}{N+1}}\right)$ secondary users are allowed to transmit, and the interference quota is on the order of $\Theta\left(n^{-\frac{1}{N+1}}\right)$. With the aforementioned choice of interference quota, or the number of allowable active users, we state one of the main results of this paper as follows.

Theorem 1: Consider a secondary MAC with an $m$-antenna base station and $n$ users each with power constraint $\rho_{s}$. The secondary MAC operates in the presence of a primary broadcast channel with an $M$-antenna transmitter with power $P_{p}$ to $N$ users each with interference tolerance $\Gamma$. The secondary throughput satisfies

$$
\begin{aligned}
\mathcal{R}_{\text {mac }} \geq \frac{m}{N+1} \log n+ & \frac{1}{N+1} \log \left(\rho_{s} \Gamma^{N}\right)-m \log \left(1+P_{p}\right) \\
& +O\left(n^{-\frac{1}{N+1}} \log n\right) \\
\mathcal{R}_{\text {mac }} \leq \frac{m}{N+1} \log n+ & \frac{1}{N+1} \log \left(\rho_{s} \Gamma^{N}\right)-\mathcal{R}_{I} \\
& +O\left(n^{-\frac{1}{N+1}}\right)
\end{aligned}
$$


with

$$
\mathcal{R}_{I}=m_{\min } \log \left(1+\frac{P_{p}}{M} \exp \left(\frac{1}{m_{\min }} \sum_{j=1}^{m_{\min }} \sum_{i=1}^{m_{\max }-j} \frac{1}{i}-\gamma\right)\right)
$$

where $m_{\min }=\min (m, M), m_{\max }=\max (m, M)$, and $\gamma$ is Euler's constant. This throughput is achieved under the threshold-based user selection with the choice of $\bar{k}_{s}$ given by (19).

\section{Proof: See Appendix I.}

Remark 2: The essence of the above result is that the secondary throughput grows as $\frac{m}{N+1} \log n+O(1)$, which implies that the secondary throughput decreases almost linearly with the number of primary constraints as $n \rightarrow \infty$. A noteworthy special case is when the primary base station chooses to transmit to a number of users equal to the number of its transmit antennas $(N=M)$, a strategy which is known to be near-optimum in terms of sum rate [18]. Under this condition

$$
\mathcal{R}_{\text {mac }}=\frac{m}{M+1} \log n+O(1)
$$

Therefore, we have

$$
\lim _{n \rightarrow \infty} \frac{\mathcal{R}_{\text {mac }}}{\mathcal{R}_{\text {mac }, w / o}^{\text {ot }}}=\frac{1}{M+1}
$$

where $\mathcal{R}_{\text {mac, } w / o}^{\text {opt }}$ is the maximum throughput of the secondary MAC in the absence of the primary system. This ratio shows that the compliance penalty of the secondary MAC system and its relationship with the characteristics of the primary network.

It is noteworthy that although $\Gamma$ does not affect the growth rate, it is an important parameter. Both the lower and upper bounds have the term $\frac{1}{N+1} \log \left(\rho_{s} \Gamma^{N}\right)=$ $\frac{1}{N+1} \log \rho_{s}+\frac{N}{N+1} \log \Gamma$, thus throughput is an increasing function of $\Gamma$. One can also see that the interference tolerance $\Gamma$ is more important than secondary power $\rho_{s}$, respectively by a factor of $\frac{N}{N+1}$ versus $\frac{1}{N+1}$.

Remark 3: The results in Theorem 1 can be directly extended to a scenario where each primary user tolerates a different level of interference. As long as all primary users allow nonzero interference (no matter how small), we can let $\Gamma$ be the minimum allowable interference, and the theorem still holds.

So far we have analyzed the effect of small but constant primary interference constraints and shown that the secondary throughput improves with increasing the number of secondary users. The flexibility provided by the increasing number of secondary users can be exploited not only to increase secondary throughput, but also to reduce the primary interference. In fact, it is possible to simultaneously suppress the interference on the primary down to zero while increasing the secondary throughput proportional to $\log n$. The following corollary makes this idea precise:

Corollary 1: Assuming the interference on each primary user is bounded as $\Theta\left(n^{-q}\right)$, the secondary throughput satisfies

$$
\mathcal{R}_{\text {mac }}=\frac{m-q N}{N+1} \log n+O(1)
$$

where $0<q<\frac{1}{N}$.
Proof: Because the proof of Theorem 1 holds for $\Gamma=$ $\Theta\left(n^{-q}\right)$, the corollary follows by substituting $\Gamma=\Theta\left(n^{-q}\right)$ into the lower and upper bounds given by Theorem 1 .

Remark 4: The corollary above explores a tradeoff where primary interference is made to decrease polynomially, i.e., proportional to $n^{-q}$. We saw that this leads to a secondary throughput that decreases linearly in $q$. If we reduce the primary interference more slowly, e.g., decreasing as $\Theta(1 / \log n)$, one can verify that $\mathcal{R}_{\text {mac }}=\frac{m}{N+1} \log n-\frac{N}{N+1} \log \log n+O(1)$, which achieves the optimal growth rate even though the throughput is reduced. Conversely, if we try to suppress the primary interference faster than $\Theta\left(n^{-1 / N}\right)$, the secondary throughput will asymptotically remain stagnant, i.e., $\mathcal{R}_{\text {mac }}=o(\log n)$, since in this case $\bar{k}_{s}=O(1)$ according to (19).

Secondary MAC With Primary MAC: Recall that each antenna at the primary base station allows interference with power $\Gamma$. By regarding each antenna of the primary base station as a virtual user, we can reuse most of the analysis that was developed in the previous section. Thus, the steps leading to (19) can be repeated to obtain the number of allowable active secondary users

$$
\bar{k}_{s}=\left(\frac{\Gamma}{\rho_{s}}\right)^{\frac{M}{M+1}}(n)^{\frac{1}{M+1}}+O(1) .
$$

With this allowable active users $\bar{k}_{s}$ and slight modifications, we obtain a result that parallels Theorem 1 .

Theorem 2: Consider a secondary MAC with an $m$-antenna base station and $n$ users each with power constraint $\rho_{s}$. The secondary MAC operates in the presence of a primary MAC channel where each user transmits with power $\rho_{p}$ to an $M$-antenna base station with interference tolerance $\Gamma$ on each antenna. The secondary throughput satisfies

$$
\begin{aligned}
\mathcal{R}_{\text {mac }} \geq & \frac{m}{M+1} \log n+\frac{1}{M+1} \log \left(\rho_{s} \Gamma^{M}\right) \\
& -m \log \left(1+\rho_{p} N\right)+O\left(n^{-\frac{1}{M+1}} \log n\right) \\
\mathcal{R}_{\text {mac }} \leq & \frac{m}{M+1} \log n+\frac{1}{M+1} \log \left(\rho_{s} \Gamma^{M}\right)-\mathcal{R}_{I} \\
& +O\left(n^{-\frac{1}{M+1}}\right)
\end{aligned}
$$

with

$$
\mathcal{R}_{I}=m_{\min } \log \left(1+\rho_{p} \exp \left(\frac{1}{m_{\min }} \sum_{j=1}^{m_{\min }} \sum_{i=1}^{m_{\max }-j} \frac{1}{i}-\gamma\right)\right)
$$

where $m_{\min }=\min (m, N), m_{\max }=\max (m, N)$, and $\gamma$ is Euler's constant. This throughput is achieved under the threshold-based user selection with the choice of $\bar{k}_{s}$ given by (25).

A tradeoff exists between the primary interference reduction and the secondary throughput enhancement, which is stated by the following corollary. Remark 4 is again applicable here.

Corollary 2: Assuming the interference on each antenna of the primary base station is bounded as $\Theta\left(n^{-q}\right)$, the secondary throughput satisfies

$$
\mathcal{R}_{\text {mac }}=\frac{m-q M}{M+1} \log n+O(1)
$$

where $0<q<\frac{1}{M}$. 


\section{Upper Bounds for Secondary Throughput}

So far we have seen achievable rates of a cognitive MAC channel in the presence of either a primary broadcast or MAC. We now develop corresponding upper bounds.

Theorem 3: Consider a secondary MAC with an $m$-antenna base station and $n$ users. The maximum throughput of the secondary, $\mathcal{R}_{\text {mac }}^{\text {opt }}$, satisfies

$$
\mathcal{R}_{\text {mac }}^{\text {opt }} \leq \frac{m}{N+1} \log n+O(\log \log n)
$$

in the presence of a primary broadcast channel transmitting to $N$ users. Similarly, $\mathcal{R}_{\text {mac }}^{\text {opt }}$ satisfies

$$
\mathcal{R}_{\text {mac }}^{\text {opt }} \leq \frac{m}{M+1} \log n+O(\log \log n)
$$

in the presence of a primary MAC, where each user transmits to an $M$-antenna base station.

Proof: See Appendix II.

Remark 5: By comparing the upper bounds with the achievable rates obtained by the thresholding strategy, we see that the achievable rates are at most $O(\log \log n)$ away from the upper bounds, a difference which is negligible relative to the dominant term $\Theta(\log n)$. Thus, the growth of the maximum throughput of a cognitive MAC is $\frac{m}{N+1} \log n$ in the presence of the primary broadcast channel, and $\frac{m}{M+1} \log n$ in the presence of the primary MAC channel. Both the achievable rates and the upper bounds show that the average cognitive sum-rate decreases almost linearly with the number of primary-imposed constraints, asymptotically.

\section{Discussion}

Recall that our method determines eligible cognitive MAC users based on their cross-channel gains. To satisfy the interference constraints, our selection rule then allows $\Theta\left(n^{\frac{1}{N+1}}\right)$, or $\Theta\left(n^{\frac{1}{M+1}}\right)$, of these users to be active simultaneously, in the presence of either the primary broadcast or MAC. If there are more eligible users than the allowed number, we choose from among the eligible users randomly. In this process, the forward channel gain of the cognitive users does not come into play, and still an optimal growth rate is achieved.

This can be intuitively explained as follows. The total received signal power at the cognitive base station grows linearly with the number of active users, and the total received signal power determines the throughput. On the other hand, selecting good cognitive users according to their secondary channel strengths can only offer logarithmic power gains (with respect to $n$ ) [10], which is negligible compared to the linear gain due to increasing the number of active users. Therefore, the cross-channel gains are more important in this case. ${ }^{5}$ Note that we do not imply that knowledge of the cognitive forward channel is useless; our conclusion only says that once the cross channels are taken into account, the asymptotic growth of the secondary throughput cannot be improved by any use of the cognitive forward channel.

\footnotetext{
${ }^{5}$ In a somewhat different context, the work of Jamal et al. [13] also indicates that cross channels can be more important than the forward channels.
}

Although we have allowed the base stations to have multiple antennas, so far the users have been assumed to have only one antenna. We now consider a generalization to the case where all users have multiple antennas. Consider a secondary MAC in the presence of a primary broadcast, where each primary and secondary user have $t_{p}$ and $t_{s}$ antennas, respectively. We apply a separate interference constraint on each antenna of each primary user, which guarantees the satisfaction of the overall interference constraint on any primary user. On each of the $t_{s}$-antenna secondary users, we shall allocate $t_{s}-1$ degrees of freedom for zero-forcing and only one degree of freedom for cognitive transmission. Using this strategy, we can ensure that $t_{s}-1$ of the primary receive antennas are exempt from interference. Thus, the total number of interference constraints will reduce from $t_{p} N$ to $t_{p} N+1-t_{s}$. By using an analysis similar to the development of Theorem 1, one can show that the growth rate $\frac{m \log n}{\max \left(1, t_{p} N+2-t_{s}\right)}$ is achievable. For the converse, the situation is more complicated, because here the correlation among the antennas of the secondary users must be accounted for. Nevertheless, in some cases, it is possible to show without much difficulty that the previously achieved throughput is indeed asymptotically optimal. For example, in the presence of the primary MAC, if $t_{s}>M$, the secondary MAC channel can have a throughput that grows as $m \log n$ by letting each active secondary user completely eliminate the interference on the primary. Similarly, in the presence of a primary broadcast channel, if $t_{s}>t_{p} N$, the secondary MAC channel can also have a throughput that grows as $m \log n$. The achieved growth rate is optimal because it coincides with the growth rate of $\mathcal{R}_{\text {mac, } w / o}^{\mathrm{opt}}$, which is always an upper bound.

\section{Cognitive Broadcast Channel}

\section{A. Achievable Scheme}

We consider a random beam-forming technique where the secondary base station opportunistically transmits to $m$ secondary users simultaneously [16]. Specifically, the secondary base station constructs $m$ orthonormal beams, denoted by $\left\{\phi_{j}\right\}_{j=1}^{m}$, and assigns each beam to a secondary user. Then, the secondary base station broadcasts to $m$ selected users. The selection of users and beam assignment will be addressed shortly.

Considering an equal power allocation among $m$ users, the transmitted signal from the secondary base station is given by

$$
\mathbf{x}_{s}=\sum_{j=1}^{m} \sqrt{\frac{P}{m}} \phi_{j} x_{j}
$$

where $\phi_{j}$ is the beam-forming vector $j$ with dimension $m \times 1$, $x_{j}$ is the signal transmitted along with the beam $j$, and $P$ is the total transmit power. In this case, we have

$$
Q_{s}=\frac{P}{m} I_{m}
$$

Notice that $P$ is subject to the power constraint $P_{s}$ as well as a set of interference constraints imposed by the primary. Thus, the value of $P$ depends on the cross channels from the secondary base station to the primary system. 
Assuming the beam $j$ is assigned to the user $i$. From (1) and (32), the received signal at the secondary user $i$ is given by

$$
y_{i}=\mathbf{h}_{i}^{\dagger} \phi_{j} x_{j}+\sum_{k \neq j} \mathbf{h}_{i}^{\dagger} \phi_{k} x_{k}+\mathbf{g}_{s, i}^{\dagger} \mathbf{x}_{p}+w_{i}
$$

where $\mathbf{h}_{i}^{\dagger}$ is the $1 \times m$ vector of channel coefficients from the secondary base station to the secondary user $i$, and $\mathbf{g}_{s, i}^{\dagger}$ is the $1 \times M($ or $1 \times N)$ vector of channel coefficients from the primary base station (or users) to the secondary user $i$. The received signal-to-noise-plus-interference-ratio (SINR) at the secondary user $i$ (with respect to the beam $j$ ) is

$$
\operatorname{SINR}_{i, j}=\frac{\frac{P}{m}\left|\mathbf{h}_{i}^{\dagger} \phi_{j}\right|^{2}}{1+\frac{P}{m} \sum_{k \neq j}\left|\mathbf{h}_{i}^{\dagger} \phi_{k}\right|^{2}+\mathbf{g}_{s, i}^{\dagger} Q_{p} \mathbf{g}_{s, i}} .
$$

The random beam technique assigns each beam to the secondary user that results in the highest SINR. Because the probability of more than two beams being assigned to the same secondary user is negligible [16], we have the secondary throughput

$$
\begin{aligned}
\mathcal{R}_{b c} & \approx \mathbb{E}\left[\sum_{j=1}^{m} \log \left(1+\max _{1 \leq i \leq n} \operatorname{SINR}_{i, j}\right)\right] \\
& =m \mathbb{E}\left[\log \left(1+\max _{1 \leq i \leq n} \operatorname{SINR}_{i, j}\right)\right] .
\end{aligned}
$$

The aforementioned analysis holds in the presence of either the primary broadcast or MAC channel; the only difference is the constraints on $P$ and $Q_{p}$. Since the SINR is symmetric across all beams, the subscript $j$ will be omitted in the following analysis.

Remark 6: We briefly address the issue of channel state information. All users are assumed to have receiver side channel state information. On the transmit side, the secondary base station only needs to know SINR and does not need to have full channel knowledge. Each secondary user can estimate its own SINR with respect to each beam, and feed it back to the secondary base station [16]. Based on collected SINR, the secondary base station performs user selection. The secondary base station needs to know $\mathbf{G}_{p}$ to adjust $P$ such that the interference constraints on the primary are satisfied.

\section{B. Throughput Calculation}

1) Secondary Broadcast With Primary Broadcast: The secondary system has to comply with the constraints on $N$ primary users. To maximize the throughput, the secondary base station transmits at the maximum allowable power. From (9) and (33), we have

$$
P=\min \left(\frac{m \Gamma}{\left|\mathbf{g}_{p, 1}^{\dagger}\right|^{2}}, \ldots, \frac{m \Gamma}{\left|\mathbf{g}_{p, N}^{\dagger}\right|^{2}}, P_{s}\right)
$$

where $\mathbf{g}_{p . \ell}^{\dagger}$ is the row $\ell$ of $\mathbf{G}_{p}$. Then, we substitute $Q_{p}$ given by (8) into (35), and obtain the SINR at the secondary user $i$ with respect to the beam $j$ :

$$
\operatorname{SINR}_{i}=\frac{\left|\mathbf{h}_{i}^{\dagger} \phi_{j}\right|^{2}}{\frac{m}{P}+\sum_{k \neq j}\left|\mathbf{h}_{i}^{\dagger} \phi_{k}\right|^{2}+\frac{m P_{p}}{M P}\left|\mathbf{g}_{s, i}\right|^{2}} .
$$

Our analysis of $\max _{i} \mathrm{SINR}_{i}$, which is required to evaluate the throughput in (37), does not follow [16] because the denominator involves a sum of two Gamma distributions with different scale parameters: $\sum_{k \neq j}\left|\mathbf{h}_{i}^{\dagger} \phi_{k}\right|^{2}$ has Gamma $(m-1,1)$ and $\frac{m P_{p}}{M P}\left|\mathbf{g}_{s, i}\right|^{2}$ has Gamma $\left(M, \frac{m P_{p}}{M P}\right)$. Fortunately, lower and upper bounds can be leveraged to simplify the analysis. We define

$$
\theta=\frac{m P_{p}}{M P}
$$

We consider the case when $\frac{m P_{p}}{M P_{s}} \geq 1$. The techniques can be generalized to the case of $\frac{m P_{p}}{M P_{s}}<1.6$ When $\frac{m P_{p}}{M P_{s}} \geq 1$, we have $\theta \geq 1$ for all $P$. We define

$$
L_{i}=\frac{\left|\mathbf{h}_{i}^{\dagger} \phi_{j}\right|^{2}}{\frac{m}{P}+\theta\left(\sum_{k \neq j}\left|\mathbf{h}_{i}^{\dagger} \phi_{k}\right|^{2}+\left|\mathbf{g}_{s, i}\right|^{2}\right)}
$$

and

$$
U_{i}=\frac{\left|\mathbf{h}_{i}^{\dagger} \phi_{j}\right|^{2}}{\frac{m}{P}+\theta\left|\mathbf{g}_{s, i}\right|^{2}}
$$

where $L_{i}$ and $U_{i}$ are random variables that depend on channel realizations. Conditioned on $P$, the denominators of $L_{i}$ and $U_{i}$ have Gamma distributions, which simplifies the analysis.

For $1 \leq i \leq n$, we have

$$
L_{i} \leq \operatorname{SINR}_{i} \leq U_{i} .
$$

Hence, for any channel realization

$$
L_{\max } \leq \max _{1 \leq i \leq n} \operatorname{SINR}_{i} \leq U_{\max }
$$

where $L_{\max }=\max _{i} L_{i}$ and $U_{\max }=\max _{i} U_{i}$. Therefore, the secondary throughput is bounded as follows:

$$
m \mathbb{E}\left[\log \left(1+L_{\max }\right)\right] \leq \mathcal{R}_{b c} \leq m \mathbb{E}\left[\log \left(1+U_{\max }\right)\right] .
$$

We study the lower and upper bounds given by (45), instead of directly analyzing $\mathcal{R}_{b c}$. Some useful properties of $L_{\max }$ and $U_{\max }$ are as follows.

Lemma 1: Conditioned on $P=\rho$

$\mathbb{P}\left(L_{\text {max }} \geq b_{n}-\frac{\rho}{m} \log \log n \mid P=\rho\right)=1-\Theta\left(\frac{1}{n}\right)$

${ }^{6}$ When $\frac{m P_{p}}{M P_{s}}<1$, one can define $\theta=\max \left(\frac{m P_{p}}{M P}, 1\right)$. Then, we can use the Bayesian expansion via conditioning on $\left\{P<\frac{m P_{p}}{M}\right\}$ and its complement, where both conditional terms can be shown to have the same growth rate. 


$$
\begin{aligned}
& \mathbb{P}\left(U_{\max }<d_{n}+\frac{\rho}{m} \log \log n \mid P=\rho\right)=1-\Theta\left(\frac{1}{\log n}\right) \\
& \mathbb{E}\left[U_{\max } \mid U_{\max }>d_{n}+\frac{\rho}{m} \log \log n, P=\rho\right]<O(n \log n)
\end{aligned}
$$

where $b_{n}=\frac{\rho}{m} \log n-\frac{\rho(m+M-1)}{m} \log \log n+O(\log \log \log n)$ and $d_{n}=\frac{\rho}{m} \log n-\frac{\rho M}{m} \log \log n+O(\log \log \log n)$.

Proof: See Appendix III.

Based on the previous two lemmas, we obtain the following results for the secondary throughput.

Theorem 4: Consider a secondary broadcast channel with $n$ users and an $m$-antenna base station with power constraint $P_{s}$. The secondary broadcast operates in the presence of a primary broadcast channel transmitting with power $P_{p}$ to $N$ users each with interference tolerance $\Gamma$. The secondary throughput satisfies

$$
\mathcal{R}_{b c}>m \log (\Gamma \log n)-m \log \left(\tilde{\mu}_{1}+\frac{m \Gamma}{P_{s}}\right)+O\left(\frac{\log \log n}{\log n}\right)
$$$$
\mathcal{R}_{b c}<m \log (\Gamma \log n)-m \log \tilde{\mu}_{2}+O(1)
$$

where $\tilde{\mu}_{1}=\mathbb{E}\left[\max _{1 \leq i \leq N}\left|\mathbf{g}_{p, i}^{\dagger}\right|^{2}\right]$ and $\tilde{\mu}_{2}=$ $\left(\mathbb{E}\left[1 / \max _{1 \leq i \leq N}\left|\mathbf{g}_{p, i}^{\dagger}\right|^{2}\right]\right)^{-1}$.

Proof: See Appendix IV.

Remark 7: The aforementioned result states that $\mathcal{R}_{b c}=$ $m \log \log n+O(1)$, thus

$$
\lim _{n \rightarrow \infty} \frac{\mathcal{R}_{b c}}{\mathcal{R}_{b c, w / o}^{\mathrm{opt}}}=1
$$

where $\mathcal{R}_{b c, w / o}^{\mathrm{opt}}$ is the maximum throughput of the secondary broadcast channel in the absence of the primary system. Therefore, the achieved throughput is asymptotically optimal, because we always have $\mathcal{R}_{b c} \leq \mathcal{R}_{b c, w / o}^{\text {opt }}$. Thus, we have a positive result: The growth rate of the secondary throughput is unaffected by the constraints and interference imposed by the primary, as long as each primary user tolerates some fixed interference $\Gamma$.

The aforementioned results naturally lead to the question: How small can we make the interference on the primary, while still having a secondary throughput that grows as $\Theta(\log \log n)$. We find that $\Gamma$, the interference on each primary user, can asymptotically go to zero, as shown by the next corollary.

Corollary 3: Assuming the interference on each primary user is bounded as $\Theta\left((\log n)^{-q}\right)$, the secondary throughput satisfies

$$
\mathcal{R}_{b c}=(1-q) m \log \log n+O(1)
$$

where $0<q<1$.

Remark 8: This result sheds lights on the tradeoff between two goals of a cognitive radio system: High throughput for the secondary and low interference on the primary. For primary interference reduction up to $\Theta\left((\log \log n)^{-1}\right)$, one can verify that $\mathcal{R}_{b c}=m \log \log n-m \log \log \log n+O(1)$, which still achieves the double logarithmic growth rate for secondary throughput. It is possible to reduce the interference faster than $\Theta\left((\log n)^{-1}\right)$, but this will make $\mathcal{R}_{b c}=o(\log \log n)$.

Remark 9: It can be shown that the growth rate of the secondary throughput does not depend on the transmit covariance $Q_{p}$ of the primary. To see this, we decompose $Q_{p}=U \Lambda U^{\dagger}$, where $U$ is an unitary matrix and $\Lambda=\operatorname{diag}\left(\lambda_{1}, \ldots, \lambda_{M}\right), 0<$ $\lambda_{1} \leq \cdots \leq \lambda_{M}<P_{p}$. From (35), we have $\mathbf{g}_{s, i}^{\dagger} Q_{p} \mathbf{g}_{s, i}=$ $\tilde{\mathbf{g}}_{s, i}^{\dagger} \Lambda \tilde{\mathbf{g}}_{s, i}$, where $\tilde{\mathbf{g}}_{s, i}=U^{\dagger} \mathbf{g}_{s, i}$ has the same distribution as $\mathbf{g}_{s, i}[19]$. Therefore, $\lambda_{1}\left|\tilde{\mathbf{g}}_{s, i}\right|^{2} \leq \mathbf{g}_{s, i}^{\dagger} Q_{p} \mathbf{g}_{s, i} \leq \lambda_{M}\left|\tilde{\mathbf{g}}_{s, i}\right|^{2}$. With the exception of a slightly different definition of $\theta$, the analysis for $Q_{p}=I$ will follow.

2) Secondary Broadcast With Primary MAC: The analysis of this case closely parallels the analysis of the primary broadcast. The secondary transmit power is given by

$$
P=\min \left(\frac{m \Gamma}{\left|\mathbf{g}_{p, 1}^{\dagger}\right|^{2}}, \ldots, \frac{m \Gamma}{\left|\mathbf{g}_{p, M}^{\dagger}\right|^{2}}, P_{s}\right)
$$

where $\mathbf{g}_{p, \ell}^{\dagger}$ is the row $\ell$ of $\mathbf{G}_{p}$. The MAC primary system produces power $N \rho_{p}$ and has $M$ interference constraints. From the viewpoint of the secondary, this is all the information that is needed. Therefore, the analysis of Theorem 4 can be essentially repeated to obtain the following result.

Theorem 5: Consider a secondary broadcast channel with $n$ users and an $m$-antenna base station with power constraint $P_{s}$. The secondary broadcast operates in the presence of a primary MAC where each user transmits with power $\rho_{p}$ to an $M$-antenna base station with interference tolerance $\Gamma$ on each antenna. The secondary throughput satisfies

$$
\begin{aligned}
& \mathcal{R}_{b c}>m \log (\Gamma \log n)-m \log \left(\tilde{\mu}_{3}+\frac{m \Gamma}{P_{s}}\right)+O\left(\frac{\log \log n}{\log n}\right) \\
& \mathcal{R}_{b c}<m \log (\Gamma \log n)-m \log \tilde{\mu}_{4}+O(1)
\end{aligned}
$$

where $\tilde{\mu}_{3}=\mathbb{E}\left[\max _{1 \leq i \leq M}\left|\mathbf{g}_{p, i}^{\dagger}\right|^{2}\right]$ and $\tilde{\mu}_{4}=$ $\left(\mathbb{E}\left[1 / \max _{1 \leq i \leq M}\left|\mathbf{g}_{p, i}^{\dagger}\right|^{2}\right]\right)^{-1}$.

Remark 10: Theorems 4 and 5 can be extended to a scenario where each primary (secondary) user has multiple antennas via regarding each primary and secondary antenna as a virtual user. Using analysis similar to the single-antenna case, the secondary broadcast channel can be shown to achieve a throughput scaling as $m \log \log n$ (thus optimal). The details are straightforward and are therefore omitted for brevity.

Similar to Corollary 3, we can also obtain the tradeoff between the primary interference reduction and the secondary throughput enhancement as follows. All the remarks following Corollary 3 apply to the present case as well.

Corollary 4: Assuming the interference on each antenna of the primary base station is bounded as $\Theta\left((\log n)^{-q}\right)$, the secondary throughput satisfies

$$
\mathcal{R}_{b c}=(1-q) m \log \log n+O(1)
$$

where $0<q<1$. 


\section{CApacity Scaling Under Path Loss and Shadowing}

The results so far were developed assuming all fading channels obey the same distribution, i.e., for a homogeneous network. In this section, we generalize our results by allowing different users to experience varying path loss and shadowing. We consider the combined effect of path loss and shadowing as a multiplicative factor on the channel gain. The probabilistic behavior of this multiplicative factor can in general be complicated because it depends on the spatial distribution of users, whose randomness will induce a distribution on path loss, as well as the composition of the terrain. However, certain assumptions can be made about it from first principles. We assume the support of the probability density of path loss and shadowing is positive $^{7}$ and bounded. This is equivalent to saying that the distance between nodes cannot be arbitrarily large or arbitrarily small, and that shadowing attenuates but is not a perfect isolator of emissions [20]. Conditioned on a realization of path loss and shadowing, the resulting fading coefficient is assumed to be a Rayleigh random variable whose variance is determined by the value of path loss and shadowing.

In this section, we concentrate on a broadcast primary. Similar results hold with little variation for the primary MAC channel and are omitted for brevity.

Our basic idea of characterizing the secondary throughput in the presence of path loss and shadowing is as follows. We find an upper (lower) bound on the secondary throughput by constructing a homogeneous network whose throughput is no larger (smaller) than the throughput under any realization of path loss and shadowing. The throughput of the homogeneous networks that bound our performance follows the analysis of previous sections. We then show the scaling of the throughput lower and upper bounds are identical.

\section{A. Secondary MAC}

A homogeneous secondary MAC channel with cross link variance $\nu$ and secondary link variance $\mu$ can be shown, using methods of the previous sections, to have a throughput characterized by

$$
\begin{gathered}
\mathcal{R}_{\text {mac }}^{\mathrm{iid}}(\nu, \mu, n) \geq \frac{m}{N+1} \log n+\frac{1}{N+1} \log \left(\rho_{s}\left(\frac{\Gamma}{\nu}\right)^{N}\right)+\log \mu \\
-m \log \left(1+P_{p}\right)+O\left(n^{-\frac{1}{N+1}} \log n\right) \\
\mathcal{R}_{\text {mac }}^{\mathrm{iid}}(\nu, \mu, n) \leq \frac{m}{N+1} \log n+\frac{1}{N+1} \log \left(\rho_{s}\left(\frac{\Gamma}{\nu}\right)^{N}\right)+\log \mu \\
-\mathcal{R}_{I}+O\left(n^{-\frac{1}{N+1}} \log n\right) .
\end{gathered}
$$

Now, consider a heterogeneous network where path loss and shadowing of the nodes vary according to a distribution with positive and bounded support. Then, conditioned on the path loss and shadowing, the channel coefficient from the secondary

\footnotetext{
${ }^{7}$ For ease of exposition, we have assumed that under path loss and shadowing a link is not completely lost. It is possible to carry through the analysis as long as at least $O(n)$ secondary links remain available, and no more than $o(\log n)$ cross links go to zero. If too many cross links disappear due to path loss and shadowing, effectively that part of the network is no longer cognitive and the nature of the problem would be changed.
}

user $i$ to the primary user $j$ is $\mathcal{C N}\left(0, \sigma_{j i}^{2}\right)$, and from the secondary user $i$ to all the co-located secondary base station antennas is $\mathcal{C N}\left(0, \sigma_{s i}^{2}\right)$, while all other channel coefficients are i.i.d. $\mathcal{C N}(0,1)$.

Let $X=\left\{\sigma_{j i}^{2}, \sigma_{s i}^{2}, 1 \leq j \leq N, 1 \leq i \leq n\right\}$, the set of all random channel variances. The positivity and boundedness assumptions for the support of the path loss distribution are formalized by $0<\nu_{1} \leq \sigma_{j i}^{2} \leq \nu_{2}$ and $0<\mu_{1} \leq \sigma_{s i}^{2} \leq \mu_{2}$.

We now outline an argument based on the intuition that the secondary throughput increases or at worst stays the same if one secondary link improves, and that the secondary throughput does not increase if one cross link gets stronger. To make this argument precise, the cross-link variance $\sigma_{j i}^{2}$ can be absorbed into the interference constraint $\Gamma$, resulting in an equivalent cross link with unit variance and interference constraint $\frac{\Gamma}{\sigma_{j i}^{2}}$. So a stronger cross link is equivalent to a stricter interference constraint, therefore the secondary throughput is nonincreasing in $\sigma_{j i}^{2}$. Similarly, the secondary link variance $\sigma_{j}^{2}$ can be absorbed into the secondary transmit power for the secondary throughput calculation, leading to an effective transmit power $\sigma_{j}^{2} \rho_{s}$ over a link of unit variance. Thus, the throughput is nondecreasing with $\sigma_{j}^{2}$. Based on the previous argument, we always have $\mathcal{R}_{\text {mac }} \geq$ $\mathcal{R}_{\text {mac }}^{\text {iid }}\left(\nu_{2}, \mu_{1}, n\right)$ and $\mathcal{R}_{\text {mac }} \leq \mathcal{R}_{\text {mac }}^{\text {iid }}\left(\nu_{1}, \mu_{2}, n\right)$, because $\nu_{1} \leq$ $\sigma_{j i}^{2} \leq \nu_{2}$ and $\mu_{1} \leq \sigma_{s i}^{2} \leq \mu_{1}$ for any realization of $X$. Therefore

$$
\mathbb{E}\left[\mathcal{R}_{\text {mac }}\right] \geq \mathcal{R}_{\text {mac }}^{\mathrm{iid}}\left(\nu_{2}, \mu_{1}, n\right)
$$

and

$$
\mathbb{E}\left[\mathcal{R}_{\text {mac }}\right] \leq \mathcal{R}_{\text {mad }}^{\mathrm{iid}}\left(\nu_{1}, \mu_{2}, n\right) .
$$

From (53), we conclude that the growth rate of our proposed technique under path loss and shadowing is $\frac{m}{N+1} \log n+O(1)$.

However, this has not fully settled the capacity question, because the upper bound was calculated only under a specific scheme. A stronger upper bound is obtained by noting that for any transmission scheme, the throughput of the heterogenous network is smaller than the throughput of the homogeneous network with cross link variance $\nu_{1}$ and secondary link variance $\mu_{2}$. The latter throughput can be shown, following the analysis of Theorem 3, to be upper bounded by $\frac{m}{N+1} \log n+$ $O(\log \log n)$.

Thus, we have lower and upper bounds whose order matches, and we have the following result.

Theorem 6: Consider a secondary MAC channel with $n$ users, $m$ antennas at the base station, and power constraint $\rho_{s}$, in the presence of a primary that broadcasts with power constraint $P_{p}$ to $N$ users with interference constraint $\Gamma$. The users are randomly located resulting in path loss and shadowing coefficients whose combined effect can be characterized by a random variable whose support is over a strictly positive bounded interval; then

$$
\begin{aligned}
\frac{m}{N+1} \log n+O(1) & \leq \mathbb{E}\left[\mathcal{R}_{\text {mac }}^{\mathrm{opt}}\right] \\
& \leq \frac{m}{N+1} \log n+O(\log \log n) .
\end{aligned}
$$

Therefore, the throughput grows with $\frac{m}{N+1} \log n$. 


\section{B. Secondary Broadcast}

Now, we consider a secondary broadcast channel. A homogeneous secondary broadcast channel with primary-to-secondary channel variance $\nu$ and secondary link variance $\mu$ can be shown to have a throughput

$$
\mathcal{R}_{b c}^{\mathrm{iid}}(\nu, \mu, n)=m \log (\mu \Gamma \log n)+O(1) .
$$

Consider the channel coefficient from all the co-located secondary base-station antennas to the secondary user $i$ to be $\mathcal{C N}\left(0, \sigma_{i}^{2}\right)$, and the primary to the secondary user $i$ to be $\mathcal{C N}\left(0, \sigma_{p i}^{2}\right)$, while all other channel coefficients are i.i.d. $\mathcal{C N}(0,1)$. Let $X=\left\{\sigma_{i}^{2}, \sigma_{p i}^{2}, 1 \leq i \leq n\right\}$, where $0<\mu_{3} \leq \sigma_{i}^{2} \leq \mu_{4}$ and $0<\nu_{3} \leq \sigma_{p i}^{2} \leq \nu_{4}$.

Similar to the argument for cognitive MAC channel, $\mathcal{R}_{b c}$ decreases with $\sigma_{p i}^{2}$ but increases with $\sigma_{i}^{2}$. Therefore, we have

$$
\mathcal{R}_{b c}^{\mathrm{iid}}\left(\nu_{4}, \mu_{3}, n\right) \leq \mathbb{E}_{X}\left[\mathcal{R}_{b c}\right] \leq \mathcal{R}_{b c}^{i i d}\left(\nu_{3}, \mu_{4}, n\right) .
$$

Thus, in the presence of path loss and shadowing, we have the following result.

Theorem 7: Consider a secondary broadcast channel with $n$ users, $m$ antennas at the base station, and power constraint $\rho_{s}$, in the presence of a primary that broadcasts with power constraint $P_{p}$ to $N$ users with interference constraint $\Gamma$. The users are randomly located resulting in path loss and shadowing coefficients whose combined effect can be characterized with a random variable whose support is over a strictly positive bounded interval; then

$$
\lim _{n \rightarrow \infty} \frac{\mathbb{E}\left[\mathcal{R}_{b c}^{\mathrm{opt}}\right]}{m \log \log n}=1
$$

Remark 11: The heterogeneity of the following channels does not affect the throughput growth rate in a straightforward manner; thus, it is not considered in the aforementioned analysis: 1) For the secondary MAC channel, the cross channel between the primary and secondary base-stations, whose variance only affects the interference on the secondary (independent of $n$ ), and 2) for the secondary broadcast channel, the cross channel from the secondary base-station to the primary, which only affects the secondary transmit power that is once again independent of $n$.

\section{NUMERICAL RESULTS}

In this section, we concentrate on numerical results in the presence of the primary broadcast channel and the results in the presence of the primary MAC channel are similar thus omitted. For all simulations, we consider $P_{p}=P_{s}=\rho_{s}=5$, the secondary base station has $m=4$ antennas, and the primary base station has $M=2$ antennas and the number of primary users is $N=2$. Fig. 3 illustrates the secondary throughput given by Theorem 1 . The allowable interference power on each primary user is $\Gamma=2$. The slope of the throughput curve is discontinuous at some points, because the allowable number of active secondary users must be an integer $\left\lfloor k_{s}\right\rfloor$. As mentioned earlier, the floor operation does not affect the asymptotic results. Fig. 4 presents the tradeoff between the tightness of the primary constraints and the secondary throughput, as shown by Corollary 1 .

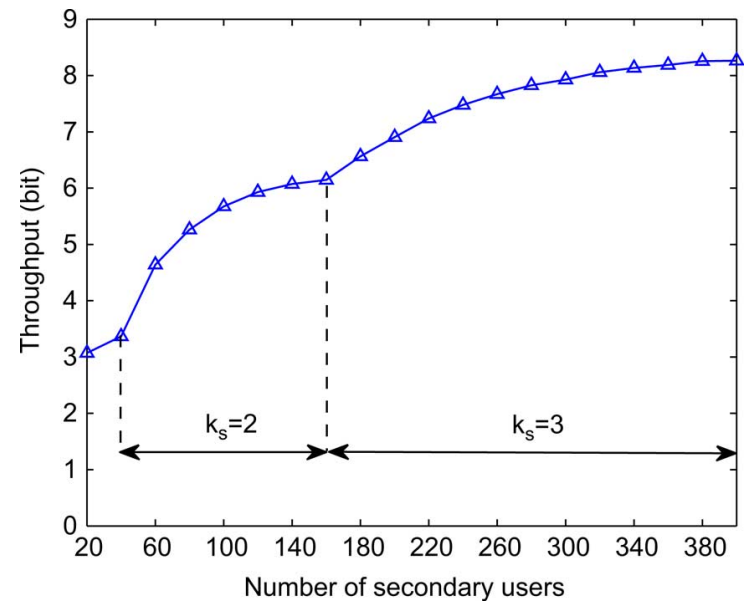

Fig. 3. Secondary MAC: Throughput versus user number $(\Gamma=2)$.
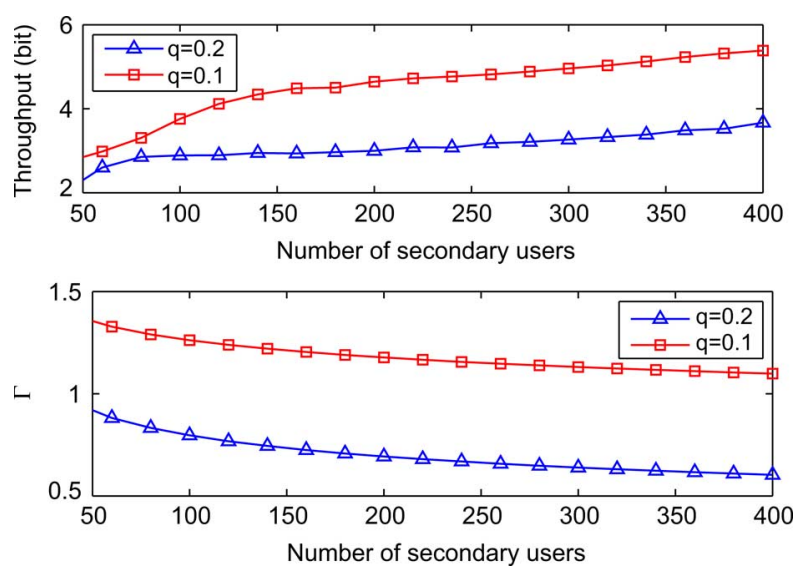

Fig. 4. Secondary MAC: Throughput versus user number $\left(\Gamma=2 n^{-q}\right)$.

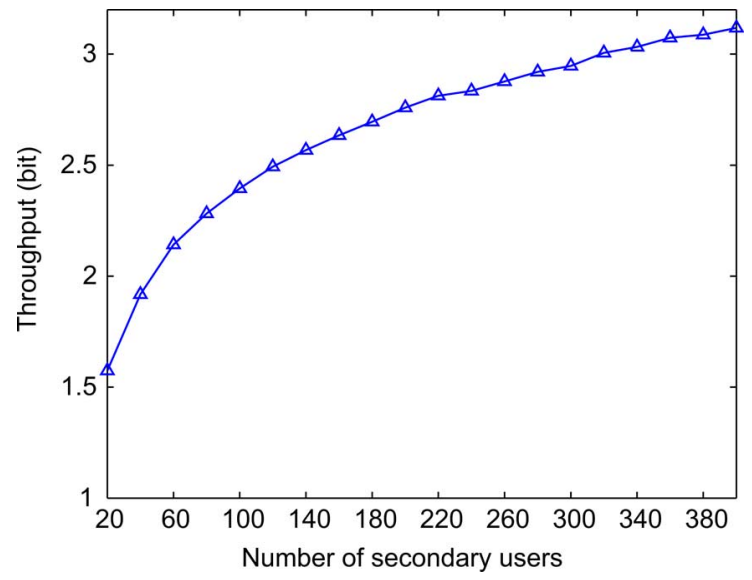

Fig. 5. Secondary broadcast: Throughput versus user number $(\Gamma=2)$.

The interference power constraint $\Gamma$ is $2 n^{-q}$ for $q=0.1$ and 0.2 , respectively. As expected, for $q=0.2$, the interference on primary decreases faster than $q=0.1$ and the secondary throughput increases more slowly.

Fig. 5 shows the secondary throughput versus the number of secondary users in the presence of the primary broadcast channel (see Theorem 4), where the interference power is $\Gamma=2$. In Fig. 6, we show the tradeoff between the secondary throughput and the interference on the primary, as described 

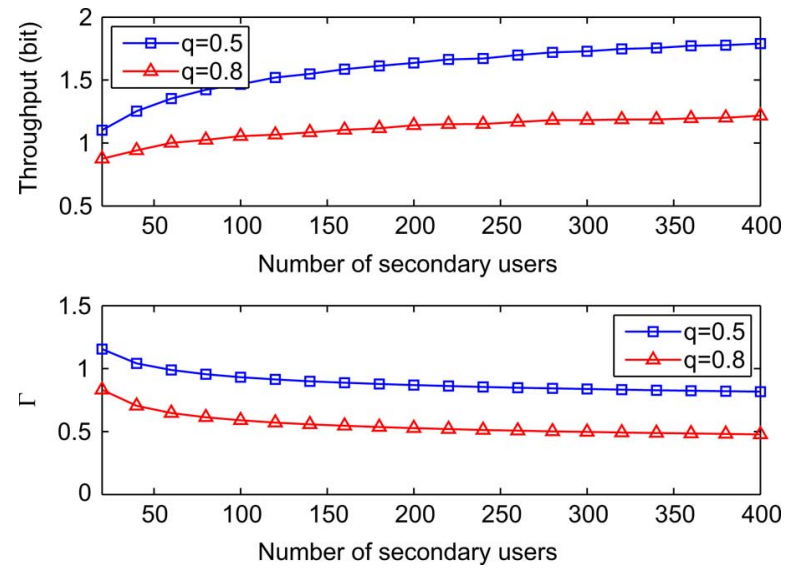

Fig. 6. Secondary broadcast: Throughput versus user number $(\Gamma=$ $\left.2(\log n)^{-q}\right)$.

in Corollary 3. We set $\Gamma$ to decline as $2(\log n)^{-q}$, for $q=0.5$ and $q=0.8$, respectively. Clearly, for $q=0.5$, the interference power decreases faster than $q=0.8$, while the secondary throughput increases more slowly.

\section{CONCLUSION}

In this paper, we study the performance limits of an underlay cognitive network consisting of a multiuser and multiantenna primary and secondary systems. We find the throughput limits of the secondary system as well as the tradeoff between this throughput and the tightness of constraints imposed by the primary system. Given a set of interference power constraints on the primary, the maximum throughput of the secondary MAC grows as $\frac{m}{N+1} \log n$ (primary broadcast), and $\frac{m}{M+1} \log n$ (primary MAC). These growth rates are attained by a simple threshold-based user selection rule. Interestingly, the secondary system can force its interference on the primary to zero while maintaining a growth rate of $\Theta(\log n)$. For the secondary broadcast channel, the secondary throughput can grow as $m \log \log n$ in the presence of either the primary broadcast or MAC channel. The growth rate of the throughput is unaffected by the presence of the primary (thus optimal). Furthermore, the interference on the primary can also be made to decline to zero, while maintaining the secondary throughput to grow as $\Theta(\log \log n)$.

\section{APPENDIX I \\ PROOF OF THEOREM 1}

Proof: We rewrite (10) as

$$
R_{\text {mac }}=\log \operatorname{det}\left(I+\mathbf{H}(\mathcal{S}) Q_{s} \mathbf{H}^{\dagger}(\mathcal{S})\left(I+\mathbf{G}_{s} Q_{p} \mathbf{G}_{s}^{\dagger}\right)^{-1}\right)
$$

The secondary throughput is calculated by averaging the instant rate $R_{\text {mac }}$ over all channel realizations, i.e., $\mathbf{H}$ and $\mathbf{G}_{s}$; thus

$$
\begin{aligned}
& \mathcal{R}_{\mathrm{mac}}= \mathbb{E}_{\mathbf{H}}\left[\mathbb{E}_{\mathbf{G}_{s}}\left[R_{\mathrm{mac}} \mid \mathbf{H}\right]\right] \\
&=\mathbb{E}_{\mathbf{H}}\left[\mathbb { E } _ { \mathbf { G } _ { s } } \left[\operatorname { l o g } \operatorname { d e t } \left(I+\mathbf{H}(\mathcal{S}) Q_{s} \mathbf{H}^{\dagger}(\mathcal{S})\right.\right.\right. \\
&\left.\left.\left.\quad \times\left(I+\mathbf{G}_{s} Q_{p} \mathbf{G}_{s}^{\dagger}\right)^{-1}\right)\right]\right] .
\end{aligned}
$$

For any positive-definite matrix $A$ and $B$, the function $\log \operatorname{det}\left(I+A B^{-1}\right)$ is convex in $B$ [21, Lemma II.3], so we apply the Jensen inequality on the right hand side of the inequality (61), i.e., taking expectation with respect to $\mathbf{G}_{s}$

$$
\begin{aligned}
& \mathcal{R}_{\text {mac }}>\mathbb{E}_{\mathbf{H}} {\left[\operatorname { l o g } \operatorname { d e t } \left(I+\mathbf{H}(\mathcal{S}) Q_{s} \mathbf{H}^{\dagger}(\mathcal{S})\right.\right.} \\
&\left.\left.\times\left(I+\mathbb{E}\left[\mathbf{G}_{s} Q_{p} \mathbf{G}_{s}^{\dagger}\right]\right)^{-1}\right)\right] \\
&=\mathbb{E}_{\mathbf{H}}\left[\log \operatorname{det}\left(I+\frac{\rho_{s}}{1+P_{p}} \mathbf{H}(\mathcal{S}) \mathbf{H}^{\dagger}(\mathcal{S})\right)\right]
\end{aligned}
$$

where in (63), we use the facts that $Q_{p}=P_{p} I / M$ and $\mathbb{E}\left[\mathbf{G}_{s} \mathbf{G}_{s}^{\dagger}\right]=M I_{m}$ since each entry of $\mathbf{G}_{s}$ is i.i.d. $\mathcal{C N}(0,1)$.

Now we bound the right-hand side of (63). Recall that $|\mathcal{A}|$ and $|\mathcal{S}|$ are the random number of eligible users and active users, respectively. By the Chebychev inequality, for any $\epsilon>0$, we have

$$
\begin{aligned}
\mathbb{P}\left(|\mathcal{A}|>(1-\epsilon) \bar{k}_{s}\right) & >1-\frac{1-p}{\epsilon^{2} n p} \\
& =1-O\left(\bar{k}_{s}^{-1}\right)
\end{aligned}
$$

where in the above, we use the fact $\bar{k}_{s}=n p$. Then, we expand (63) based the event $\left\{|\mathcal{A}|>(1-\epsilon) \bar{k}_{s}\right\}$ and its complement, and discard the nonnegative term associated with its complement

$$
\begin{gathered}
\mathcal{R}_{\text {mac }} \\
>\mathbb{E}\left[\log \operatorname{det}\left(I+\frac{\rho_{s}}{1+P_{p}} \mathbf{H}(\mathcal{S}) \mathbf{H}^{\dagger}(\mathcal{S})\right)|| \mathcal{A} \mid>(1-\epsilon) \bar{k}_{s}\right] \\
\times \mathbb{P}\left(|\mathcal{A}|>(1-\epsilon) \bar{k}_{s}\right) \\
\geq \mathbb{E}\left[\log \operatorname{det}\left(I+\frac{\rho_{s}}{1+P_{p}} \mathbf{H}(\mathcal{S}) \mathbf{H}^{\dagger}(\mathcal{S})\right)|| \mathcal{A} \mid=(1-\epsilon) \bar{k}_{s}\right] \\
=\mathbb{E}\left[\log \operatorname{det}\left(I+\frac{\rho_{s}}{1+P_{p}} \mathbf{H}(\mathcal{S}) \mathbf{H}^{\dagger}(\mathcal{S})\right) \mid\right. \\
\times\left(1-O\left(\bar{k}_{s}^{-1}\right)\right)
\end{gathered}
$$

where in the inequality (67), we apply the result in (65) and the fact that the conditional expectation of the right hand side of (66) is nondecreasing in $|\mathcal{A}|$. Since $|\mathcal{S}|=(1-\epsilon) \bar{k}_{s}$ in case of $|\mathcal{A}|=(1-\epsilon) \bar{k}_{s}$, then we obtain (68) due to the throughput depending on $|\mathcal{A}|$ via the size of $\mathcal{S}$.

Recall that each entry of $\mathbf{H}(\mathcal{S})$ is i.i.d. $\mathcal{C N}(0,1)$. Conditioned on $|\mathcal{S}|=(1-\epsilon) \bar{k}_{s}, \mathbf{H}(\mathcal{S}) \mathbf{H}^{\dagger}(\mathcal{S})$ is a Wishart Matrix with degrees of freedom $(1-\epsilon) \bar{k}_{s}$, we have [22, Lemma A]

$$
\begin{array}{r}
\mathcal{R}_{\text {mac }}>\left(m \log \left(1+\frac{(1-\epsilon) \rho_{s} \bar{k}_{s}}{1+P_{p}}\right)+O\left(\bar{k}_{s}^{-1}\right)\right) \\
\times\left(1-O\left(\bar{k}_{s}^{-1}\right)\right) \\
=m \log \left(1+P_{p}+(1-\epsilon) \rho_{s} \bar{k}_{s}\right)+O\left(\frac{\log \bar{k}_{s}}{\bar{k}_{s}}\right) \\
-m \log \left(1+P_{p}\right) \\
=m \log \rho_{s} \bar{k}_{s}+m \log (1-\epsilon)-m \log \left(1+P_{p}\right) \\
+O\left(\frac{\log \bar{k}_{s}}{\bar{k}_{s}}\right)
\end{array}
$$


where in (71), we use the identity $\log (x+y)=\log x+\log (1+$ $y / x)$, for $x, y>0$. Since the strict inequality (71) holds for any $\epsilon>0$, thus $\log (1-\epsilon)<0$ but can be arbitrarily close to zero, by the definition of inequality, we have

$$
\mathcal{R}_{\text {mac }} \geq m \log \rho_{s} \bar{k}_{s}-m \log \left(1+P_{p}\right)+O\left(\frac{\log \bar{k}_{s}}{\bar{k}_{s}}\right) .
$$

Now we find an upper bound for $\mathcal{R}_{\text {mac }}$. For convenience, we denote [see (10)]

$$
R_{\text {mac }, 0}=\log \operatorname{det}\left(I+\rho_{s} \mathbf{H}(\mathcal{S}) \mathbf{H}^{\dagger}(\mathcal{S})+\mathbf{G}_{s} Q_{p} \mathbf{G}_{s}^{\dagger}\right)
$$

and

$$
R_{I}=\log \operatorname{det}\left(I+\mathbf{G}_{s} Q_{p} \mathbf{G}_{s}^{\dagger}\right)
$$

So the throughput can be written as

$$
\mathcal{R}_{\text {mac }}=\mathbb{E}\left[R_{\text {mac }, 0}\right]-\mathbb{E}\left[R_{I}\right] .
$$

Using the inequality $\operatorname{det}(A) \leq(\operatorname{tr}(A) / k))^{k}[23$, p. 680], where $A$ is a $k \times k$ positive-definite matrix, $R_{\text {mac }, 0}$ is bounded by

$R_{\text {mac }, 0} \leq m \log \left(1+\frac{1}{m} \operatorname{tr}\left(\rho_{s} \mathbf{H}(\mathcal{S}) \mathbf{H}^{\dagger}(\mathcal{S})+\mathbf{G}_{s} Q_{p} \mathbf{G}_{s}^{\dagger}\right)\right)$.

Therefore

$$
\begin{aligned}
\mathbb{E}\left[R_{\mathrm{mac}, 0}\right] \leq & m \mathbb{E}\left[\operatorname { l o g } \left(1+\frac{1}{m} \operatorname{tr}\left(\rho_{s} \mathbf{H}(\mathcal{S}) \mathbf{H}^{\dagger}(\mathcal{S})\right.\right.\right. \\
& \left.\left.\left.+\mathbf{G}_{s} Q_{p} \mathbf{G}_{s}^{\dagger}\right)\right)\right] \\
\leq & m \log \left(1+\frac{\rho_{s}}{m} \mathbb{E}\left[\operatorname{tr}\left(\mathbf{H}(\mathcal{S}) \mathbf{H}^{\dagger}(\mathcal{S})\right)\right]\right. \\
& \left.+\frac{1}{m} \mathbb{E}\left[\operatorname{tr}\left(\mathbf{G}_{s} Q_{p} \mathbf{G}_{s}^{\dagger}\right)\right]\right) \\
\leq & m \log \left(1+\rho_{s} \bar{k}_{s}+P_{p}\right)
\end{aligned}
$$

where (78) uses the Jensen inequality. To obtain inequality (79), we use the facts that $\mathbb{E}\left[\operatorname{tr}\left(\mathbf{G}_{s} Q_{p} \mathbf{G}_{s}^{\dagger}\right)\right]=P_{p}$ by substituting $Q_{p}$ given by $(8)$ as well as $\mathbb{E}\left[\operatorname{tr}\left(\mathbf{H}(\mathcal{S}) \mathbf{H}^{\dagger}(\mathcal{S})\right)\right] \leq m \bar{k}_{s}$ due to $|\mathcal{S}| \leq \bar{k}_{s}$.

Now we lower bound the second term in (75). From [24, Th. 1], we have

$$
\begin{aligned}
\mathbb{E}\left[R_{I}\right] & \geq m_{\min } \log \left(1+\frac{P_{p}}{M} \exp \left(\frac{1}{m_{\min }} \sum_{j=1}^{m_{\min }} \sum_{i=1}^{m_{\max }-j} \frac{1}{i}-\gamma\right)\right) \\
& \triangleq \mathcal{R}_{I}
\end{aligned}
$$

where $m_{\min }=\min (m, M), m_{\max }=\max (m, M)$, and $\gamma$ is the Euler's constant. Notice that $\mathcal{R}_{I}$ is a finite constant independent of $n$ and $\Gamma$.

Combining (79) and (80), we have

$$
\mathcal{R}_{\text {mac }} \leq m \log \left(1+\rho_{s} \bar{k}_{s}+P_{p}\right)-\mathcal{R}_{I}
$$

Finally, substituting $\bar{k}_{s}$ given by (19) and noting that $\bar{k}_{s}=$ $\Theta\left(n^{\frac{1}{N+1}}\right)$, we have

$$
\begin{aligned}
\mathcal{R}_{\text {mac }} \geq & \frac{m}{N+1} \log n+\frac{1}{N+1} \log \left(\rho_{s} \Gamma^{N}\right) \\
& -m \log \left(1+P_{p}\right)+O\left(n^{-\frac{1}{N+1}} \log n\right) \\
\mathcal{R}_{\text {mac }} \leq & \frac{m}{N+1} \log n+\frac{1}{N+1} \log \left(\rho_{s} \Gamma^{N}\right) \\
& -\mathcal{R}_{I}+O\left(n^{-\frac{1}{N+1}}\right)
\end{aligned}
$$

where we use the identity $\log (x+y)=\log x+\log (1+y / x)$, for $x, y>0$, in the aforementioned inequalities. This completes the proof.

Remark 12: The primary transmit covariance matrix $Q_{p}$ can be arbitrary and does not affect the growth rate of $\mathcal{R}_{\text {mac }}$. For any $Q_{p}$, we have $Q_{p}=U \Lambda U^{\dagger}$, where $U$ is an unitary and $\Lambda=\operatorname{diag}\left[\lambda_{1}, \ldots, \lambda_{M}\right]$. For the lower bound, in (62) we have $\mathbb{E}\left[\mathbf{G}_{s} Q_{p} \mathbf{G}_{s}^{\dagger}\right]=\mathbb{E}\left[\mathbf{G}_{s} U \Lambda U^{\dagger} \mathbf{G}_{s}^{\dagger}\right]=\mathbb{E}\left[\mathbf{G}_{s 1} \Lambda \mathbf{G}_{s 1}^{\dagger}\right]$, where each entry of $\mathbf{G}_{s 1}$ is still i.i.d. $\mathcal{C N}(0,1)$ [19]. Let $\mathbf{g}_{i}$ be the column $i$ of $\mathbf{G}_{s 1}$, then $\mathbb{E}\left[\mathbf{G}_{s} Q_{p} \mathbf{G}_{s}^{\dagger}\right]=\mathbb{E}\left[\sum_{i=1}^{M} \lambda_{i} \mathbf{g}_{i} \mathbf{g}_{i}^{\dagger}\right]=\sum_{i=1}^{M} \lambda_{i} I_{m}$. Since $\operatorname{tr}\left(Q_{p}\right)=P_{p}, \sum_{i=1}^{M} \lambda_{i}=P_{p}$, which yields the same bound as (63), and the same development of the lower bound. For the upper bound, we note that in (78) $\mathbb{E}\left[\operatorname{tr}\left(\mathbf{G}_{s} Q_{p} \mathbf{G}_{s}^{\dagger}\right)\right]=$ $\operatorname{tr}\left(\mathbb{E}\left[\mathbf{G}_{s 1} \Lambda \mathbf{G}_{s 1}^{\dagger}\right]\right)=\sum_{i=1}^{M} \lambda_{i} \operatorname{tr}\left(I_{m}\right)=m P_{p}$, which yields the same bound (79) and thus the development of the upper bound.

\section{APPENDIX II \\ ProOF OF THEOREM 3}

Proof: We develop an upper bound for the secondary throughput in the presence of the primary broadcast only; the development is similar in the presence of the primary MAC and thus is omitted. We consider an arbitrary active user set $\mathcal{S}$ and transmit covariance matrix given by (4), such that the interference constraints on the primary are satisfied.

By removing the interference from the primary to the secondary, the secondary throughput is enlarged. Then, starting from (10) and using the inequality $\operatorname{det}(A) \leq(\operatorname{tr}(A) / k)^{k}[23$, p. 680], where $A_{k \times k}$ is a positive-definite matrix, we have

$$
R_{\mathrm{mac}} \leq m \log \left(1+\frac{1}{m} \operatorname{tr}\left(\mathbf{H}(\mathcal{S}) Q_{s} \mathbf{H}^{\dagger}(\mathcal{S})\right)\right)
$$

Let $\mathbf{h}_{i}$ be the $m \times 1$ vector of channel coefficients from the secondary user $i(i \in \mathcal{S})$ to the secondary base station, corresponding to a certain column of $\mathbf{H}(\mathcal{S})$. Since $Q_{s}$ is diagonal, we have

$$
\operatorname{tr}\left(\mathbf{H}(\mathcal{S}) Q_{s} \mathbf{H}^{\dagger}(\mathcal{S})\right)=\sum_{i \in \mathcal{S}} \rho_{i} \operatorname{tr}\left(\mathbf{h}_{i} \mathbf{h}_{i}^{\dagger}\right)
$$




$$
\begin{aligned}
& =\sum_{i \in \mathcal{S}} \rho_{i}\left|\mathbf{h}_{i}\right|^{2} \\
& \leq \max _{i \in \mathcal{S}}\left|\mathbf{h}_{i}\right|^{2} \sum_{i \in \mathcal{S}} \rho_{i} \\
& \leq \max _{1 \leq i \leq n}\left|\mathbf{h}_{i}\right|^{2} \sum_{i \in \mathcal{S}} \rho_{i}
\end{aligned}
$$

where $\rho_{i}$ is the transmit power of the secondary user $i$. Let

$$
P_{\text {sum }}=\sum_{i \in \mathcal{S}} \rho_{i}
$$

and

$$
h_{\max }=\max _{1 \leq i \leq n}\left|\mathbf{h}_{i}\right|^{2} .
$$

We can rewrite the right-hand side of (84) as

$$
R_{\text {mac }} \leq m \log \left(1+\frac{1}{m} h_{\text {max }} P_{\text {sum }}\right) .
$$

We first bound $P_{\text {sum }}$ and formulate an optimization as

$$
\max _{\mathcal{S},\left\{\rho_{i}\right\}} P_{\text {sum }}
$$

subject to $\rho_{i} \leq \rho_{s}$ for $i \in \mathcal{S}$

$$
\left[\mathbf{G}_{p} Q_{s} \mathbf{G}_{p}^{\dagger}\right]_{\ell, \ell} \leq \Gamma \text { for } 1 \leq \ell \leq N
$$

which is a standard linear programming, and the solution is denoted by $P_{\text {sum }}^{*}$. Then, $P_{\text {sum }}^{*}$ is the maximum total transmit power, depending on the channel realizations for each transmission.

Subject to the interference constraints on the primary, the user selection and power allocation are coupled, and a direct analysis is difficult. Instead, we will find an upper bound for $P_{\text {sum }}^{*}$. Notice that the total interference (on all primary users) caused by the secondary user $i$ is $\rho_{i}\left|\mathbf{g}_{p, i}\right|^{2}$, where $\mathbf{g}_{p, i}$ is the vector of channel coefficients from the secondary $i$ to all $N$ primary users. We relax the set of individual interference constraints in (92) with a single sum interference constraint

$$
\sum_{i \in \mathcal{S}} \rho_{i}\left|\mathbf{g}_{p, i}\right|^{2} \leq N \Gamma
$$

Notice that $\mathbf{g}_{p, i}$ corresponds to a certain column in $\mathbf{G}_{p}$.

Order the cross-channel gains $\left\{\left|\mathbf{g}_{p, i}\right|^{2}\right\}_{i=1}^{n}$ of all the secondary users and denote the ordered cross-channel gains by

$$
\left|\tilde{\mathbf{g}}_{p, 1}\right|^{2} \leq\left|\tilde{\mathbf{g}}_{p, 2}\right|^{2} \leq \cdots \leq\left|\tilde{\mathbf{g}}_{p, n}\right|^{2}
$$

Then, we further relax the sum interference constraint (93) by replacing $\left\{\left|\mathbf{g}_{p, i}\right|^{2}\right\}_{i \in \mathcal{S}}$ with the first $|\mathcal{S}|$ smallest cross-channel gains $\left\{\left|\tilde{\mathbf{g}}_{p, i}\right|^{2}\right\}_{i=1}^{|\mathcal{S}|}$. Thus, we have

$$
\begin{gathered}
\max _{\mathcal{S},\left\{\rho_{i}\right\}} P_{\text {sum }} \\
\text { subject to } \sum_{i=1}^{|\mathcal{S}|} \rho_{i}\left|\tilde{\mathbf{g}}_{p, i}\right|^{2} \leq N \Gamma \\
\rho_{i} \leq \rho_{s} \text { for } 1 \leq i \leq|\mathcal{S}| .
\end{gathered}
$$

For any channel realization, the solution for the aforementioned problem, denoted by $P_{\text {sum, }, 1}^{*}$, is always greater than, or equal to $P_{\text {sum }}^{*}$. Notice that $P_{\text {sum }, 1}^{*}$ is also a random variable. Since $\left\{\left|\tilde{\mathbf{g}}_{p, i}\right|^{2}\right\}$ is nondecreasing in $i$, the set of $\left\{\rho_{i}\right\}$ that achieves $P_{\text {sum }, 1}^{*}$ satisfies $\rho_{i} \geq \rho_{j}$, for $i \leq j$. In other words, we have $\rho_{i}=\rho_{s}$, for $i=1$ to $|\mathcal{S}|-1$, and $\rho_{i} \leq \rho_{s}$, for $i=|\mathcal{S}|$.

Let $S_{\max }$ be the maximum value of $|\mathcal{S}|$ that satisfies the constraint

$$
\rho_{s} \sum_{i=1}^{|\mathcal{S}|-1}\left|\tilde{\mathbf{g}}_{p, i}\right|^{2} \leq N \Gamma
$$

We have

$$
P_{\text {sum }, 1}^{*} \leq \rho_{s} S_{\max }
$$

where in (97), we have an inequality because the constraint (96) is relaxed by discarding $\rho_{|\mathcal{S}|}$ compared to the interference constraint in (95).

Now, we focus on bounding $\rho_{s} S_{\max }$. For any positive integer $k$, we have

$$
\mathbb{P}\left(S_{\max }<k\right) \geq \mathbb{P}\left(\sum_{i=1}^{k-1}\left|\tilde{\mathbf{g}}_{p, i}\right|^{2}>\frac{N \Gamma}{\rho_{s}}\right)
$$

which comes from the fact that the event of the right hand side implies the event of the left hand side. Notice that $\sum_{i=1}^{k-1}\left|\tilde{\mathbf{g}}_{p, i}\right|^{2}$ is a sum of least order statistics out of $\left\{\left|\mathbf{g}_{p, i}\right|^{2}\right\}_{i=1}^{n}$ with i.i.d. Gamma $(N, 1)$ distributions. We apply some results in the development of [13, Proposition 12], and obtain ${ }^{8}$

$$
\mathbb{P}\left(\sum_{i=1}^{f(n)-1}\left|\tilde{\mathbf{g}}_{p, i}\right|^{2}>\frac{N \Gamma}{\rho_{s}}\right)>1-O\left(\frac{1}{f(n)}\right)
$$

where $f(n)=c_{0} n^{\frac{1}{N+1}}$, and $c_{0}=\left(\frac{\Gamma(N+1)}{(1-\epsilon) \rho_{s}} N^{-\frac{1}{N}}\right)^{\frac{N}{N+1}}$. For large $N$ and small $\epsilon, c_{0} \approx \frac{\Gamma}{\rho_{s}}(N+1)$.

Let $k=f(n)$ in (98) and combine with (99):

$$
\mathbb{P}\left(\rho_{s} S_{\max }<\rho_{s} f(n)\right)>1-O\left(n^{-\frac{1}{N+1}}\right) .
$$

After characterizing $\rho_{s} S_{\max }$, now we return to $P_{\text {sum }}^{*}$. To simplify notation, we denote

$$
\bar{p}_{\text {sum }}=\rho_{s} f(n) .
$$

Because $P_{\text {sum }}^{*} \leq P_{\text {sum }, 1}^{*} \leq \rho_{s} S_{\max }$ for any channel realizations, from (100), we have

$$
\begin{aligned}
\mathbb{P}\left(P_{\text {sum }}^{*} \geq \bar{p}_{\text {sum }}\right) & =1-\mathbb{P}\left(P_{\text {sum }}^{*}<\bar{p}_{\text {sum }}\right) \\
& <1-\mathbb{P}\left(\rho_{s} S_{\text {max }}<\bar{p}_{\text {sum }}\right) \\
& <O\left(n^{-\frac{1}{N+1}}\right) .
\end{aligned}
$$

Now, we complete the analysis of $P_{\mathrm{sum}}^{*}$, and move to $h_{\max }$. Because $\left\{\left|\mathbf{h}_{i}\right|^{2}\right\}_{i=1}^{n}$ have i.i.d. Gamma $(m, 1)$ distributions, using the similar arguments developed in Lemma 1, we obtain

$$
\mathbb{P}\left(h_{\max }>\zeta_{n}\right)=O\left(\frac{1}{\log n}\right)
$$

${ }^{8}$ For our case, $\frac{1}{\lambda}=\gamma=N$. 


$$
\mathbb{E}\left[h_{\max } \mid h_{\max }>\zeta_{n}\right]<O(n \log n)
$$

where $\zeta_{n}$ is a deterministic sequence satisfying

$$
\zeta_{n}=\log n+m \log \log n+O(\log \log \log n) .
$$

Now we are ready to develop the upper bound for the secondary throughput. Since $P_{\text {sum }} \leq P_{\text {sum }}^{*}$, from (91), we have

$$
\begin{aligned}
& \mathcal{R}_{\text {mac }} \leq m \mathbb{E}_{\mathbf{H}, P}\left[\log \left(1+\frac{1}{m} h_{\max } P_{\text {sum }}^{*}\right)\right] \\
&= m \mathbb{E}_{\mathbf{H}, P}\left[\log \left(1+\frac{1}{m} h_{\max } P_{\text {sum }}^{*}\right) \mid P_{\text {sum }}^{*}<\bar{p}_{\text {sum }}\right] \\
& \times \mathbb{P}\left(P_{\text {sum }}^{*}<\bar{p}_{\text {sum }}\right) \\
&+ m \mathbb{E}_{\mathbf{H}, P}\left[\log \left(1+\frac{1}{m} h_{\max } P_{\text {sum }}^{*}\right) \mid P_{\text {sum }}^{*} \geq \bar{p}_{\text {sum }}\right] \\
& \times \mathbb{P}\left(P_{\text {sum }}^{*} \geq \bar{p}_{\text {sum }}\right) \\
& \leq m \mathbb{E}_{\mathbf{H}}\left[\log \left(1+\frac{1}{m} h_{\max } \bar{p}_{\text {sum }}\right)\right] \cdot 1 \\
&+m \mathbb{E}_{\mathbf{H}}\left[\log \left(1+\frac{1}{m} h_{\max } \rho_{s} n\right)\right] \cdot O\left(n^{-\frac{1}{N+1}}\right) \\
& \leq m \mathbb{E}_{\mathbf{H}}\left[\log \left(1+\frac{1}{m} h_{\max } \bar{p}_{\text {sum }}\right) \mid h_{\max } \leq \zeta_{n}\right] \\
& \times \mathbb{P}\left(h_{\max } \leq \zeta_{n}\right) \\
&+m \mathbb{E}_{\mathbf{H}}\left[\log \left(1+\frac{1}{m} h_{\max } \bar{p}_{\text {sum }}\right) \mid h_{\max }>\zeta_{n}\right] \\
& \times \mathbb{P}\left(h_{\max }>\zeta_{n}\right) \\
&+m \mathbb{E}_{\mathbf{H}}\left[\log \left(1+\frac{1}{m} h_{\max } \rho_{s} n\right) \mid h_{\max } \leq \zeta_{n}\right] \\
& \times \mathbb{P}\left(h_{\max } \leq \zeta_{n}\right) O\left(n^{-\frac{1}{N+1}}\right) \\
&+m \mathbb{E}_{\mathbf{H}}\left[\log \left(1+\frac{1}{m} h_{\max } \rho_{s} n\right) \mid h_{\max }>\zeta_{n}\right] \\
& \times \mathbb{P}\left(h_{\max }>\zeta_{n}\right) O\left(n^{-\frac{1}{N+1}}\right)
\end{aligned}
$$

$$
\begin{aligned}
& \leq m \log \left(1+\frac{1}{m} \zeta_{n} \bar{p}_{\text {sum }}\right) \cdot 1 \\
&+m \log \left(1+\frac{\bar{p}_{\text {sum }}}{m} \mathbb{E}\left[h_{\max } \mid h_{\max }>\zeta_{n}\right]\right) \\
& \times \mathbb{P}\left(h_{\max }>\zeta_{n}\right) \\
&+m \log \left(1+\frac{1}{m} \zeta_{n} \rho_{s} n\right) \cdot 1 \cdot O\left(n^{\left.-\frac{1}{N+1}\right)}\right. \\
&+m \log \left(1+\frac{\rho_{s} n}{m} \mathbb{E}\left[h_{\max } \mid h_{\max }>\zeta_{n}\right]\right) \\
& \times \mathbb{P}\left(h_{\max }>\zeta_{n}\right) O\left(n^{-\frac{1}{N+1}}\right)
\end{aligned}
$$

$$
\begin{aligned}
\leq & m \log \left(1+\frac{1}{m} \zeta_{n} \bar{p}_{\text {sum }}\right) \\
& +m \log \left(1+\frac{\bar{p}_{\text {sum }}}{m} O(n \log n)\right) O\left(\frac{1}{\log n}\right) \\
& +m \log \left(1+\frac{1}{m} \zeta_{n} \rho_{s} n\right) O\left(n^{-\frac{1}{N+1}}\right)
\end{aligned}
$$

$$
+m \log \left(1+\frac{\rho_{s} n}{m} O(n \log n)\right) O\left(\frac{1}{\log n}\right) O\left(n^{-\frac{1}{N+1}}\right)
$$

where the second term in (108) comes from using (102) as well as the fact that $P_{\text {sum }}^{*}$ is upper bounded by $\rho_{s} n$. In (110), we apply the Jensen inequality to obtain the second and fourth terms. Using (103) and (104), we have the second and fourth terms in (111). Finally, by substituting $\bar{p}_{\text {sum }}$ and $\zeta_{n}$, we obtain

$$
\mathcal{R}_{\text {mac }} \leq \frac{m}{N+1} \log n+O(\log \log n) .
$$

This concludes the proof of this theorem.

\section{APPENDIX III}

Proof OF LEMMA 1

Proof: First, we prove (46). Let $Z=\left|\mathbf{h}_{i}^{\dagger} \phi_{j}\right|^{2}$ and $Y=$ $\theta\left(\sum_{k \neq j}\left|\mathbf{h}_{i}^{\dagger} \phi_{j}\right|^{2}+\left|\mathbf{g}_{s, i}\right|^{2}\right)$. Then, $Z$ has the exponential distribution, and $Y$ has the Gamma $((m+M-1), \theta)$ distribution. We can write

$$
L_{i}=\frac{Z}{c+Y}
$$

where $c=\frac{m}{\rho}$. Conditioned on $Y$, the probability density function (pdf) of $L_{i}$ is given by

$$
\begin{aligned}
f_{L}(x) & =\int_{0}^{\infty} f_{L \mid Y}(x \mid y) f_{Y}(y) d y \\
& =\int_{0}^{\infty}(c+y) e^{-(c+y) x} \times \frac{y^{m+M-1} e^{-y / \theta}}{(m+M-1) ! \theta^{m+M}} d y \\
& =\frac{e^{-c x}}{(1+\theta x)^{m+M}}(c(1+\theta x)+\theta(m+M-1)) .
\end{aligned}
$$

So the cdf of $L_{i}$ is

$$
\begin{aligned}
F_{L}(x) & =1-\int_{x}^{\infty} f_{L}(t) d t \\
& =1-\frac{e^{-c x}}{(1+\theta x)^{m+M-1}} .
\end{aligned}
$$

We define a growth function as

$$
\begin{aligned}
g_{L}(x) & =\frac{1-F_{L}(x)}{f_{L}(x)} \\
& =\frac{1+\theta x}{c(1+\theta x)+\theta(m+M-1)} .
\end{aligned}
$$

Since $\lim _{x \rightarrow \infty} g_{L}^{\prime}(x)=0$, the limiting distribution of $L_{\max }=$ $\max _{1 \leq i \leq n} L_{i}$ exists [25]

$$
\lim _{n \rightarrow \infty}\left(F_{L}\left(b_{n}+a_{n} x\right)\right)^{n}=e^{-e^{-x}}
$$


where $b_{n}=F_{L}^{-1}(1-1 / n)$ and $a_{n}=g_{L}\left(b_{n}\right)$. In general, an exact closed-form solution for $a_{n}$ and $b_{n}$ is intractable, but an approximation can be obtained, which is sufficient for asymptotic analysis. After manipulating (118), we have

$$
b_{n}=\frac{1}{c} \log n-\frac{m+M-1}{c} \log \log n+O(\log \log \log n)
$$

and thus

$$
a_{n}=\frac{1}{c}+O\left(\frac{1}{\log n}\right)
$$

It is straightforward to verify $\lim _{n \rightarrow \infty}\left(n g_{L}^{\prime}\left(b_{n}\right)\right)=\infty$, so we apply the expansion developed in [26], (22)

$$
\left(F_{L}\left(b_{n}+a_{n} x\right)\right)^{n}=\exp \left(-\exp \left(-x+\Theta\left(\frac{x^{2}}{\log ^{2} n}\right)\right)\right) .
$$

Let $x=-\log \log n$ in (124); we obtain (46).

Now, we prove (47) and (48). Since $U_{i}$ is similar to $L_{i}$, except that the denominator now has the Gamma $(M, \theta)$ distribution. Following the same steps of obtaining (124), we have the expansion of the cdf of $U_{\max }$ :

$$
\left(F_{U}\left(d_{n}+c_{n} x\right)\right)^{n}=\exp \left(-\exp \left(-x+\Theta\left(\frac{x^{2}}{\log ^{2} n}\right)\right)\right)
$$

where

$$
d_{n}=\frac{1}{c} \log n-\frac{M}{c} \log \log n+O(\log \log \log n)
$$

and

$$
c_{n}=\frac{1}{c}+O\left(\frac{1}{\log n}\right)
$$

where (47) follows by substituting $x=\log \log n$ into (125).

Finally, because $\mathbb{E}\left[U_{\text {max }}\right]<n \mathbb{E}\left[U_{i}\right][25]$, we have

$$
\begin{aligned}
& \mathbb{E}\left[U_{\max } \mid U_{\max }>d_{n}+\frac{1}{c} \log \log n\right] \\
& \leq \frac{n \mathbb{E}\left[U_{i}\right]}{\mathbb{P}\left(U_{\max }>d_{n}+\frac{1}{c} \log \log n\right)}=\Theta(n \log n)
\end{aligned}
$$

where we use (47) in the last equality.

\section{APPENDIX IV}

\section{PROOF OF THEOREM 4}

Proof: We first find a lower bound for the secondary throughput $\mathcal{R}_{b c}$. Conditioned on $P=\rho$, the throughput is denoted $\mathcal{R}_{b c \mid P}(\rho)$. Let $l_{n}=b_{n}-\frac{\rho}{m} \log \log n$, where $b_{n}$ is given by Lemma 1. Using (45), the conditional throughput $\mathcal{R}_{b c \mid P}(\rho)$ can be bounded as

$$
\begin{aligned}
& \mathcal{R}_{b c \mid P}(\rho) \geq m \mathbb{E}\left[\log \left(1+L_{\max }\right) \mid P=\rho\right] \\
& \geq m \mathbb{E}\left[\log \left(1+L_{\max }\right) \mid L_{\max } \geq l_{n}, P=\rho\right] \\
& \times \mathbb{P}\left(L_{\max } \geq l_{n} \mid P=\rho\right)
\end{aligned}
$$

$$
\begin{array}{r}
>m\left(\log \left(\frac{\rho}{m} \log n\right)+O\left(\frac{\log \log n}{\log n}\right)\right) \\
\times\left(1-\Theta\left(n^{-1}\right)\right) \\
=m \log \left(\frac{\rho}{m} \log n\right)+O\left(\frac{\log \log n}{\log n}\right) .
\end{array}
$$

From (129) to (130), we discard the nonnegative term associated with the event $\left\{L_{\max }<l_{n}\right\}$. Using (46) from Lemma 1 and the identity $\log (x+y)=\log x+\log (1+y / x)$, for $x, y>0$, we have (131).

Now we take the expectation with respect to $P$. From (38), we have

$$
P>\frac{m \Gamma}{\max _{1 \leq i \leq N}\left|\mathbf{g}_{p, i}^{\dagger}\right|^{2}+m \Gamma / P_{s}}
$$

where $\mathbf{g}_{p, i}^{\dagger}$ is the $1 \times m$ vector of channel coefficients from the secondary base station to the primary user $i$. Let the pdf of $\max _{1 \leq i \leq N}\left|\mathbf{g}_{p}(i)\right|^{2}$ be $f_{g_{p}}(x)$. Because (133) holds for any channel realization, we have

$$
\begin{aligned}
& \mathcal{R}_{b c}>\int_{0}^{\infty} m \log \left(\frac{\Gamma \log n}{x+m \Gamma / P_{s}}\right) f_{g_{p}}(x) d x \\
&+O\left(\frac{\log \log n}{\log n}\right) \\
& \geq m \log \left(\frac{\Gamma \log n}{\tilde{\mu}_{1}+m \Gamma / P_{s}}\right)+ O\left(\frac{\log \log n}{\log n}\right) \\
&=m \log (\Gamma \log n)-m \log \left(\tilde{\mu}_{1}+m \Gamma / P_{s}\right) \\
&+O\left(\frac{\log \log n}{\log n}\right)
\end{aligned}
$$

where (135) comes from the convexity of $\log \left(a+\frac{b}{x+c}\right)$ and

$$
\tilde{\mu}_{1}=\mathbb{E}\left[\max _{1 \leq i \leq N}\left|\mathbf{g}_{p}(i)\right|^{2}\right] .
$$

To find an upper bound, we still begin with the conditional throughput $\mathcal{R}_{b c \mid P}(\rho)$. Let $u_{n}=d_{n}+\frac{\rho}{m} \log \log n$, where $d_{n}$ is given by Lemma 1 . Then

$$
\begin{aligned}
\mathcal{R}_{b c \mid}(\rho) \leq & m \mathbb{E}\left[\log \left(1+U_{\max }\right) \mid P=\rho\right] \\
\leq & m \mathbb{E}\left[\log \left(1+U_{\max }\right) \mid U_{\max }<u_{n}, P=\rho\right] \\
& \times \mathbb{P}\left(U_{\max }<u_{n} \mid P=\rho\right) \\
& +m \mathbb{E}\left[\log \left(1+U_{\max }\right) \mid U_{\max } \geq u_{n}, P=\rho\right] \\
& \times \mathbb{P}\left(U_{\max } \geq u_{n} \mid P=\rho\right) \\
< & m \log \left(1+u_{n}\right)\left(1-\Theta\left(\frac{1}{\log n}\right)\right) \\
& +m \log \left(1+\mathbb{E}\left[U_{\max } \mid U_{\max } \geq u_{n}, P=\rho\right]\right) \\
& \times \Theta\left(\frac{1}{\log n}\right) \\
< & m \log \left(1+\frac{\rho}{m} \log n\right)+O(1)
\end{aligned}
$$


where (138) comes from (45). We apply (47) in Lemma 1 and the Jensen inequality to obtain (141). Using (48) in Lemma 1 and substituting $u_{n}$, we obtain (142).

After calculating an upper bound for the conditional throughput, we average over $P$. From (38), we have

$$
P \leq \frac{m \Gamma}{\max _{1 \leq i \leq N}\left|\mathbf{g}_{p, i}^{\dagger}\right|^{2}} .
$$

We denote

$$
\frac{1}{\tilde{\mu}_{2}}=\mathbb{E}\left[1 / \max _{1 \leq i \leq N}\left|\mathbf{g}_{p, i}^{\dagger}\right|^{2}\right] .
$$

Then, by the Jensen inequality, we have

$$
\begin{aligned}
\mathcal{R}_{b c} & <m \log \left(1+\frac{\log n}{m} \mathbb{E}[P]\right)+O(1) \\
& <m \log \left(1+\frac{\Gamma}{\tilde{\mu}_{2}} \log n\right)+O(1) \\
& =m \log (\Gamma \log n)-m \log \tilde{\mu}_{2}+O(1)
\end{aligned}
$$

where (146) holds since $\mathbb{E}[P] \leq \frac{m \Gamma}{\tilde{\mu}_{2}}$. The theorem follows.

\section{ACKNOWLEDGMENT}

The authors acknowledge constructive comments from the reviewers, specifically with respect to the question of unequal path loss and shadowing. The authors acknowledge the associate editor for his meticulous editing and insightful comments that led to many improvements.

\section{REFERENCES}

[1] Facilitating opportunities for flexible, efficient, and reliable spectrum use employing cognitive radio technologies. Washington, DC, Tech. Rep. FCC-05-57A1, Dec. 2003, Federal Communications Commission.

[2] S. A. Jafar, S. Srinivasa, I. Maric, and A. Goldsmith, "Breaking spectrum gridlock with cognitive radios: An information theoretic perspective," Proc. IEEE, vol. 97, no. 5, pp. 894-914, May 2009.

[3] N. Devroye, P. Mitran, and V. Tarokh, "Achievable rates in cognitive radio channels," IEEE Trans. Inf. Theory, vol. 52, no. 5, pp. 1813-1827, May 2006.

[4] J. Mitola, "Cognitive radio: An integrated agent architecture for software defined radio," Ph.D. dissertation, KTH, Stockholm, Sweden, Dec. 2000.

[5] A. Sahai, N. Hoven, and R. Tandra, "Some fundamental limits on cognitive radio," in Proc. Allerton Conf. Commun., Control, Comput., Oct. 2004.

[6] A. Ghasemi and E. S. Sousa, "Fundamental limits of spectrum-sharing in fading environments," IEEE Trans. Wireless Commun., vol. 6, no. 2, pp. 649-658, Feb. 2007.

[7] R. Zhang and Y.-C. Liang, "Exploiting multi-antennas for opportunistic spectrum sharing in cognitive radio networks," IEEE J. Sel. Topics Signal Process., vol. 2, no. 1, pp. 88-102, Feb. 2008.

[8] R. Zhang, S. Cui, and Y.-C. Liang, "On ergodic sum capacity of fading cognitive multiple-access and broadcast channels," IEEE Trans. Inf. Theory, vol. 55, no. 11, pp. 5161-5178, Nov. 2009.

[9] M. Gastpar, "On capacity under receive and spatial spectrum-sharing constraints," IEEE Trans. Inf. Theory, vol. 53, no. 2, pp. 471-487, Feb. 2007.

[10] P. Viswanath, D. N. C. Tse, and R. Laroia, "Opportunistic beamforming using dumb antennas," IEEE Trans. Inf. Theory, vol. 48, no. 6, pp. 1277-1294, Jun. 2002.
[11] K. Hamdi, W. Zhang, and K. B. Letaief, "Opportunistic spectrum sharing in cognitive MIMO wireless networks," IEEE Trans. Wireless Commun, vol. 8, no. 8, pp. 4098-4109, Aug. 2009.

[12] N. Jamal, H. E. Saffar, and P. Mitran, "Throughput enhancements in point-to-multipoint cognitive systems," in Proc. IEEE Int. Symp. Inf. Theory, Jun./Jul. 2009, pp. 2742-2746.

[13] N. Jamal, H. E. Saffar, and P. Mitran, "Asymptotic scheduling gains in point-to-multipoint cognitive networks," 2010 [Online]. Available: http://arxiv.org/pdf/1001.3365

[14] R. J. Serfling, Approximation Theorems of Mathematical Statistics. New York: Wiley, 1980.

[15] E. Telatar, "Capacity of multi-antenna Gaussian channels," Eur. Trans. Telecomm, vol. 10, no. 6, pp. 585-595, 1999.

[16] M. Sharif and B. Hassibi, "On the capacity of MIMO broadcast channels with partial side information," IEEE Trans. Inf. Theory, vol. 51, no. 2, pp. 506-522, Feb. 2005.

[17] A. Moustakas, S. Simon, and A. Sengupta, "MIMO capacity through correlated channels in the presence of correlated interferers and noise: a (not so) large n analysis," IEEE Trans. Inf. Theory, vol. 49, no. 10 , pp. 2545-2561, Oct. 2003.

[18] G. Caire and S. Shamai, "On the achievable throughput of a multiantenna Gaussian broadcast channel," IEEE Trans. Inf. Theory, vol. 49, no. 7, pp. 1691-1706, Jul. 2003.

[19] T. L. Marzetta and B. M. Hochwald, "Capacity of a mobile multipleantenna communication link in Rayleigh flat fading," IEEE Trans. Inf. Theory, vol. 45, no. 1, pp. 139-157, Jan. 1999.

[20] H. Bolcskei, R. Nabar, O. Oyman, and A. Paulraj, "Capacity scaling laws in MIMO relay networks," IEEE Trans. Wireless Commun, vol. 5, no. 6, pp. 1433-1444, Jun. 2006.

[21] S. Diggavi and T. Cover, "The worst additive noise under a covariance constraint," IEEE Trans. Inf. Theory, vol. 47, no. 7, pp. 3072-3081, Nov. 2001.

[22] B. Hochwald, T. Marzetta, and V. Tarokh, "Multiple-antenna channel hardening and its implications for rate feedback and scheduling," IEEE Trans. Inf. Theory, vol. 50, no. 9, pp. 1893-1909, Sep. 2004.

[23] T. M. Cover and J. A. Thomas, Elements of Information Theory, 2nd ed. New York: Wiley, 2006

[24] O. Oyman, R. Nabar, H. Bolcskei, and A. Paulraj, "Characterizing the statistical properties of mutual information in MIMO channels," IEEE Trans. Signal Process, vol. 51, no. 11, pp. 2784-2795, Nov. 2003.

[25] H. A. David and H. N. Nagaraja, Order Statistics. : Wiley, 2003.

[26] N. T. Uzgoren, "The asymptotic development of the distribution of the extreme values of a sample," in Studies in Mathematics and Mechanics: Presented to Richard von Mises by Friends, Colleagues, and Pupils. New York: Academic, 1954, pp. 346-353.

Yang $\mathbf{L i}\left(\mathrm{S}^{\prime} 10\right)$ received his B.S. and M.S. degree in electrical engineering from Shanghai Jiao Tong University, Shanghai, China in 2005 and 2008, respectively. $\mathrm{He}$ is currently pursuing the Ph.D. degree in electrical engineering at the University of Texas at Dallas. He has interned at Samsung Telecommunications America in 2012, and at Huawei Technologies Co. Ltd. in 2011 and 2008. His current interests include cognitive radio, heterogeneous network, interference management and cooperative communication.

Aria Nosratinia (S'87-M'97-SM'04-F'10) is Jonsson Distinguished Professor of Engineering at the University of Texas at Dallas. He received his $\mathrm{Ph} . \mathrm{D}$. in Electrical and Computer Engineering from the University of Illinois at Urbana-Champaign in 1996. He has held visiting appointments at Princeton University, Rice University, and UCLA. His interests lie in the broad area of information theory and signal processing, with applications in wireless communications. He was the secretary for the IEEE Information Theory Society in 2010-2011 and was the treasurer for ISIT 2010 in Austin, Texas. He has served as editor for the IEEE Transactions on Information Theory, IEEE Transactions on Wireless Communications, IEEE Signal Processing Letters, IEEE Transactions on Image Processing, and IEEE Wireless Communications (Magazine). He has been the recipient of the National Science Foundation career award, and is a fellow of IEEE. 